\title{
Prevalence and Associated Factors of Intimate Partner Violence During Pregnancy in Eastern Ethiopia
}

This article was published in the following Dove Press journal: International Journal of Women's Health

\author{
Wondimye Ashenafi (D) \\ Bezatu Mengistie (iD ${ }^{2}$ \\ Gudina Egata' \\ Yemane Berhane iD $^{3}$ \\ 'School of Public Health, College of \\ Health and Medical Sciences, Haramaya \\ University, Harar, Ethiopia; ${ }^{2}$ Department \\ of Environmental Health Science, College \\ of Health and Medical Sciences, \\ Haramaya University, Harar, Ethiopia; \\ ${ }^{3}$ Department of Epidemiology, Addis \\ Continental Institute of Public Health, \\ Addis Ababa, Ethiopia
}

Background: Intimate partner violence is a major public health problem and a gross violation of human rights. The consequences of this violation are severe during pregnancy as the fetus/newborn could also be affected negatively. The aim of this study was to assess the prevalence and associated factors of intimate partner violence during pregnancy (IPVP) in Eastern Ethiopia.

Methods: A community-based cross-sectional study was conducted among 3015 postpartum mothers in Eastern Ethiopia. Data were collected using the WHO Violence Against Women questionnaire. Factors associated with IPVP were assessed by a log-binomial regression model using Stata version 14.

Results: The overall prevalence of IPVP was 30.5\% (95\% CI: 28.8, 32.1); the prevalence for psychological violence was $24.4 \%$ (95\% CI: $22.9,26.0)$, physical violence was $11.9 \%$ (95\% CI: $10.8,13.2)$ and sexual violence was $11.0 \%(95 \%$ CI: $9.9,12.2)$. About $95 \%$ of acts of sexual violence were in the form of forced sex. In multivariable analysis, women's education was associated with a decreased prevalence of all forms of IPVP. Previous experience of infant loss and not drinking alcohol were associated with lower prevalence of all IPVP types, except for the physical form. Working for cash and having medium household decision-making autonomy increased the prevalence of all forms of IPVP. Being older, living in an extended family, husband's/partner's habitual khat chewing and discordant pregnancy intentions increased the prevalence ratio (PR) of all forms of IPVP, except for sexual violence. Compared to being urban, being rural decreased the PR of sexual IPVP by $59 \%$ (adjusted prevalence ratio $[\mathrm{APR}]=0.41 ; 95 \% \mathrm{CI}: 0.27,0.60)$ and psychological IPVP by $32 \%(\mathrm{APR}=0.68 ; 95 \% \mathrm{CI}: 0.53,0.87)$. Husband's/partner's support for antenatal care use decreased the PR of physical IPVP by $56 \%$ (APR $=0.44 ; 95 \%$ CI: $0.25,0.78$ ).

Conclusion: Nearly one-third of pregnant women experienced IPVP. The great majority of them were subjected to forced sex by their husband/partner. This calls for urgent attention at all levels of societal organization, requiring stakeholders and policy makers to tackle the situation.

Keywords: intimate partner violence, pregnancy, women, abuse, Ethiopia

\section{Background}

The World Health Organization (WHO) defines intimate partner violence (IPV) against women as behavior within an intimate relationship that causes harm, including physical aggression, sexual coercion, psychological abuse and controlling behaviors by a current or former male intimate partner. ${ }^{1}$ It is considered as a gross violation of human rights. ${ }^{2}$ Violence against women can happen at any time in a woman's life, including during pregnancy. ${ }^{3}$
Correspondence: Wondimye Ashenaf School of Public Health, College of Health and Medical Sciences, Haramaya

University, Harar, Ethiopia

Email wondtg@gmail.com 
Several studies have indicated that pregnancy increases the risk of IPV. ${ }^{4-7}$ The increased risk of IPV has been found to be associated with ambivalent feelings about the pregnancy and unborn child, mounting economic pressures on the family, and decreased physical and emotional availability due to the pregnancy. ${ }^{4,6,8}$ Other studies suggest that pregnancy may also be protective against IPV if both members of the couple share the excitement and joy of becoming pregnant, and that the excitement increases the husband's biological and social drives to become supportive. ${ }^{9,10}$

The burden of intimate partner violence during pregnancy (IPVP) was estimated to vary between $1 \%$ and $28 \%$ globally. ${ }^{11}$ An average global prevalence of $17 \%$ for sexual IPVP was also reported in a meta-analysis and a higher prevalence is commonly reported in developing countries.12 In Africa, the overall prevalence of IPVP ranges between $2.3 \%$ and $57.1 \%$; the commonest form is psychological violence (2-49\%), followed by physical (23-40\%) and sexual violence (3-27\%). ${ }^{13}$ A systematic review of studies on IPVP in Ethiopia indicated a $26.1 \%$ overall prevalence, with the highest prevalence reported in Oromia regional state $(35 \%){ }^{14}$

IPVP is increasingly recognized both as a major public health problem and as an issue of gross human rights violation owing to the severe health consequences for the mother, fetus and newborn. ${ }^{15}$ The adverse health effects for the mother include injuries and chronic pain, sexually transmitted infections, traumatic fistulae, pelvic inflammatory diseases and poor general health; ${ }^{16-18}$ mental health disorders such as maternal depression, anxiety and suicidal tendencies; ${ }^{19,20}$ no or inadequate use of maternal healthcare services, ${ }^{21,22}$ and poor parenting. ${ }^{23}$ Poor pregnancy/ birth outcomes, including fetal loss, low birth weight, premature birth and newborn death, have also been linked to IPVP. ${ }^{24-26}$

As described in an ecological framework on violence against women, the occurrence of IPV against women is influenced by several factors, which are diverse and basically interconnected at individual and relationship levels. ${ }^{27}$ Most of the prior studies in Ethiopia identified individual characteristics of the women and their intimate partners, including low level of education, being a young age, early age at marriage, alcohol abuse, being a full-time housewife and living in rural areas, as contributors to IPVP. ${ }^{28-32}$ Some studies have also highlighted relationship factors, such as male dominance in economic and decision-making autonomy in the family, as well as supportive attitudes towards wife-beating, as precursors of IPVP. . $^{28,33,34}$ Pregnancy-related factors, including attendance at antenatal care (ANC), pregnancy being planned or not, and history of previous pregnancy outcomes, were also indicated in different studies in Ethiopia. ${ }^{3234-37}$

Most of the previous studies in Ethiopia were conducted in clinical settings. ${ }^{28,3032-37}$ The others were conducted in the community, in small samples of currently pregnant women ${ }^{29,31}$, or general population of women asking about abuse during past pregnancies, ${ }^{11,38}$ and were therefore prone to recall bias. Little is known about the magnitude of and the risk factors associated with the occurrence of different forms of IPVP (physical, sexual and psychological) in Ethiopia. Therefore, this study aimed (1) to estimate the prevalence of the different forms of IPVP (physical, sexual, psychological and overall IPVP), and (2) to identify factors associated with IPVP in Eastern Ethiopia.

\section{Methods}

\section{Study Design and Setting}

A community-based cross-sectional study design was used. The study was conducted in both rural (Kersa district) and urban (Harar town) populations in Eastern Ethiopia. The study sites run a health and demographic surveillance system (HDSS). The Kersa HDSS, which is predominantly rural, has 24 randomly selected kebeles (the smallest administrative unit in Ethiopia) with an estimated 127,000 inhabitants. The Harar HDSS, which is predominantly urban, has 12 kebeles with an estimated 56,000 inhabitants. Trade is the main livelihood of the people in Harar, whereas farming is the major source of livelihood for people in Kersa district. Khat (Catha edulis), a stimulant green leaf, constitutes a significant share of the trade and farming in Eastern Ethiopia. Khat chewing is prevalent in both Harar and Kersa communities. ${ }^{39}$ The study was conducted between January 2018 and October 2018.

\section{Study Population and Recruitment}

The study population comprised currently partnered mothers within the first 6 weeks of birth who were permanently residing in either the rural Kersa or urban Harar HDSS study site. A mother was considered currently partnered if she was either living together with her husband (formally married) or living together with an intimate partner in an informal marriage, at least during the index pregnancy. We excluded pregnant women not in any type 
of union because pregnancy might be from forced or voluntary casual sexual relationships.

As a first procedure, a house-to-house census was carried out to identify all pregnant women using a pregnancy-screening questionnaire and procedure, as described by Assefa et al. ${ }^{40}$ The pregnancy screening was carried out initially for all women and then at an interval of 3 months for those who were not pregnant at the initial screening. During the study period, a total of 3425 women were identified as being pregnant (Figure 1).

We calculated the expected date of delivery for all pregnant women in the study based on the women's responses. Every pregnant woman was visited repeatedly through to the end of pregnancy (to determine the outcome of the pregnancy) and, when the outcome was known to be a live birth, until the sixth week postpartum to administer the survey questionnaire. As this study was part of another study that assessed the relationship between IPVP and neonatal mortality in the same population, women who had live births were considered for interview. Thus, a final sample of 3015 currently partnered postpartum mothers who had live births were included in or considered for this study, as shown in Figure 1.

\section{Data Collection and Management}

The WHO multi-country study questionnaire, which was used previously in various studies in Ethiopia, was used to elicit necessary information on IPVP. ${ }^{11}$ The English version of the instrument was translated into local languages (Amharic and Afan Oromo) by fluent speakers of the languages and backtranslated to English by another person to ensure consistency and accuracy.

Data were collected by trained female data collectors and field supervisors; all had at least secondary school level education. We used only female data collectors and supervisors to enhance disclosure of the IPVP experience, which is in accordance with the WHO ethical safety recommendations. ${ }^{41}$ The field staff were given 1

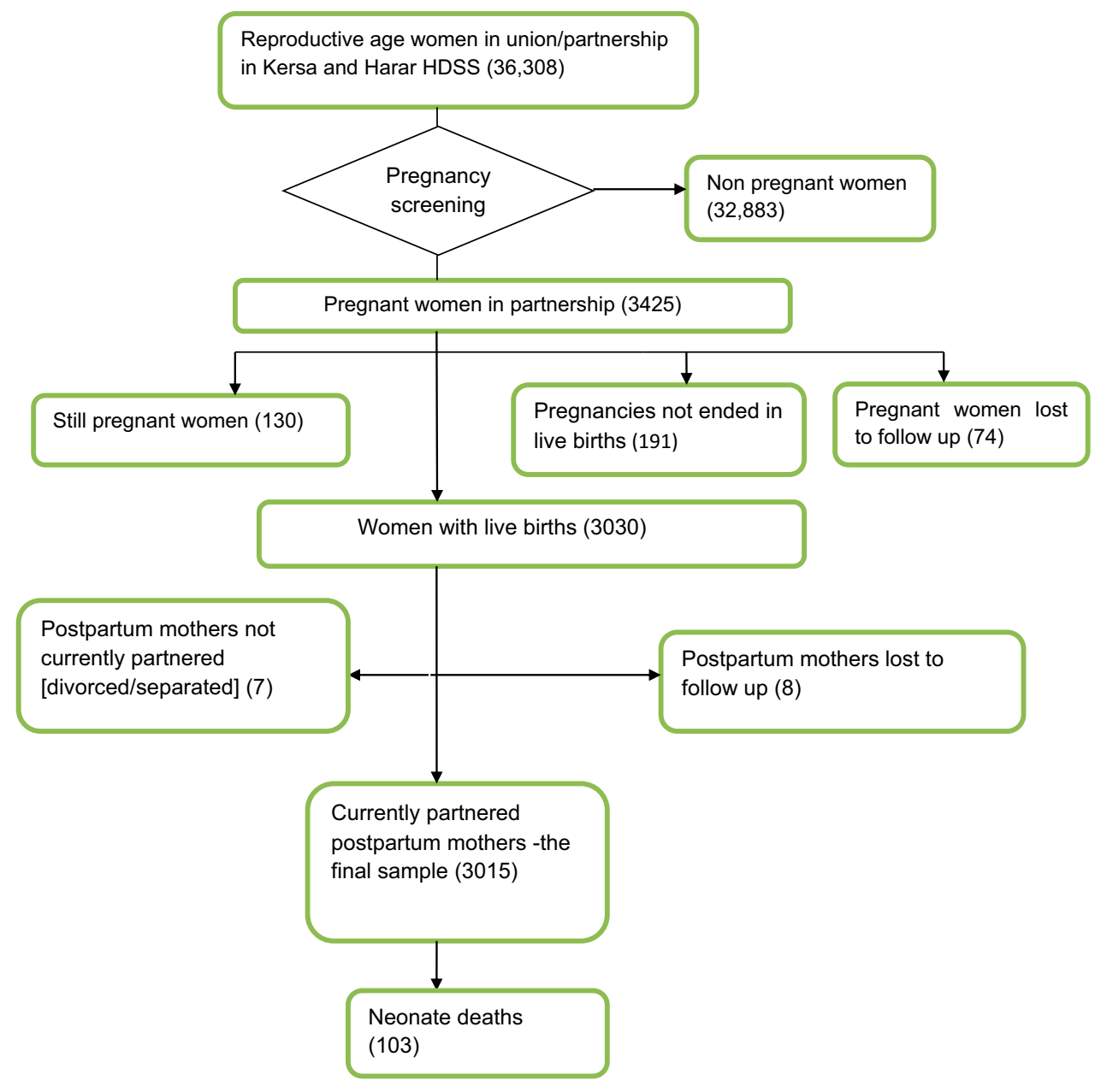

Figure I Flow diagram showing identification of study subjects, Eastern Ethiopia, 2018. 
week of intensive training based on the WHO's guide for training on domestic violence. ${ }^{42}$ The training was focused on how to administer the questionnaire, ways of addressing questions that could arise during the interview, how to maximize disclosure of violence, and safety and confidentiality. A standard fieldwork manual developed by the WHO for use in studies on violence against womenwas adopted and used by the research team. ${ }^{43}$ Before commencement of the main study, the study tool and procedure were pretested in a sample of postpartum women in similar settings outside the study area.

\section{Ethical Approval}

This study was conducted following the principles of the Declaration of Helsinki. The Institutional Health Research Ethics Review Committee of Haramaya University, College of Health and Medical Sciences, ethically cleared the study (IHRERC/153/2017, dated October 26, 2017). Written Informed consent was obtained from each participant before the interview. Minor participants (ie, those aged 15-17 years) were asked about their willingness to participate. For those willing to participate, written informed consent was obtained from their parents or legal guardians. Additional assent was then obtained from each of these minor participants. For illiterate participants, a literate witness was involved in the consent process, the participant's fingerprint was taken, and the consent form was signed by the witness and the data collector.

This study followed the WHO ethical and safety recommendation guideline for studies on violence against women. ${ }^{41}$ Thus, the study was framed as a women's health, family relationship and life experiences study. However, each participating woman was personally introduced to the specific nature of the study before the interview was conducted (through a participant information and consent acquisition sheet). The interview with each woman was conducted in a private setting and at a preferable time and place for her. The respondent was also allowed to reschedule (or relocate) the interview to a time (or place) that may be safer or more convenient for her. No identifying information was recorded on the questionnaires; instead, a unique code was used to distinguish the questionnaires. Each study subject was informed of the aim and purpose of the study, the risks and benefits of participating in the study, and that her participation was purely voluntary. Confidentiality was maintained throughout the study period.

\section{Variables and Measurement}

The experience of IPVP was considered as an outcome variable and assessed using the WHO multi-country study questionnaire. ${ }^{11}$ It was here defined as a woman's exposure to psychological, physical or sexual violence by her husband/intimate partner during the time of the index pregnancy. Our sample is comprised of mothers currently partnered with the father of the index child, so for the purposes of this study, "intimate partner" refers to the father of the respondent's index child.

Data were collected on three forms of IPVP, ie, physical, sexual and psychological.

An affirmative answer to any one of the following six behaviors constituted physical IPVP: (1) pushing or throwing something at her; (2) slapping; (3) punching or hitting with a fist or something harmful; (4) kicking or dragging her; (5) choking or burning intentionally; or (6) threatening or attacking with a weapon (eg, a knife or gun).

Sexual IPVP was measured by at least one positive response to the following three acts: (1) if the woman reported having been physically forced to have sexual intercourse when she did not want to; (2) when the woman had intercourse out of fear of her husband/partner; or (3) when she was forced to perform other sexual acts that she found degrading or humiliating.

Psychological IPVP was measured by at least one positive response to the following four questions, which asked whether the respondent's husband/partner had: (1) insulted her or made her feel bad about herself; (2) humiliated or belittled her in front of others; (3) scared/ intimidated her on purpose; or (4) threatened to hurt her or someone close to her.

Consequently, women who answered "yes" to at least one of the 13 questions above were considered as having "experienced any violence (any/overall IPVP)" and were coded as " 1 ", and women who answered "no" to all of the questions were considered as "no violence experienced" and coded as " 0 ".

Data were also collected on the socio-demographic and pregnancy-related characteristics of each respondent woman and her husband/partner. Education was grouped into no formal education, primary (Grade 1-8), secondary (Grade 9-12) and tertiary level (Grade 12+). Respondent's age was recategorized into 19 years or younger, 20-34 years and 35 years or older. Respondent's working status was categorized as housewife versus working to earn cash, whereas the husband's/partner's working status was 
grouped into working and not working. Living arrangement was grouped into living in a separate arrangement or living together with parents/families. The alcohol drinking habits of the woman and her husband/partner during the pregnancy period were classified as frequent drinker, occasional drinker and never drunk alcohol. The husband's/ partner's khat-chewing habit was grouped into never or occasional chewer versus habitual (frequent) chewer.

Women's household empowerment was derived from their household decision-making autonomy and their belief in and attitude towards wife-beating. ${ }^{44}$ Household decision making was determined based on the number of household decisions a woman made alone or jointly with her husband/partner about: (1) spending income; (2) obtaining health care for herself; (3) major household purchases; (4) purchases for daily household needs; and (5) visiting family or relatives. The response options were: (a) respondent alone, (b) respondent and husband/partner, (c) husband/partner alone, or (d) others/someone else. Each question was assigned a value of 1 if the response was (a) or (b), and 0 for (c) or (d). Attitude towards wifebeating was measured as a composite index consisting of the following items: a husband/partner is justified in hitting or beating his wife if she (a) is unable to carry household chores, (b) disobeys/argues with him, (c) asks him about his extramarital affairs, (d) is barren, and (e) refuses to have sexual intercourse with him. Each item had a response of "Yes" or "No", and a value of " 0 " was given if the woman accepted/justified beating and " 1 " otherwise.

For information related to the index pregnancy, women were asked whether they received ANC or not, their husband's/partner's stance on women's ANC use (discourage, no interest, encourage), whether there was a previous history of infant death, the number of living children and the couple's intention for the index pregnancy (concordant versus discordant intentions). The number of living children was recategorized as three or fewer, four to six, and seven or more children. Intentions regarding the index pregnancy were assessed by asking, "Right before you became pregnant, did you want to become pregnant then, did you want to wait until later, did you not want it at all?" Women were asked the same question about their husband's/partner's intentions of the index pregnancy. The index pregnancy intentions by the women and by their husband/partner were used to construct pregnancy intention discordance status for the couples. Thus, a couple's discordance was defined as a non-match between the woman's and her husband's/partner's intentions (ie, not both "wanted then", both "wanted later" or both "not wanted"), whereas concordance was defined as a match in the intention of the index pregnancy between the woman and her partner.

\section{Data Management and Analysis}

The data were double entered and cleaned using EpiData version 3.1 and analyzed using Stata version 14. A principal component analysis (PCA) was performed to derive the women's household empowerment index using each of the variables representing women's household decisionmaking autonomy and their belief in/attitude towards wife-beating. The PCA yielded three categories of women's household empowerment: low, medium and high status. In the analysis, the correlation coefficients between the components and original variable were used to understand the structure of latent factors in the model.

A log-binomial regression model was used to assess factors associated with the outcome variable. The logbinomial regression model is an appropriate analytical model for estimating the prevalence ratio (PR) in crosssectional studies of binary and common outcomes. ${ }^{45}$

Crude prevalence ratios (CPRs) with $95 \%$ CIs were estimated to assess the association between each independent variable and the outcome variable. Variables with a $P$ value $<0.25$ in the bivariate analysis were first considered for multivariable analysis; however, known confounders such as residence, living arrangement and the number of children under 5 years of age that were deemed to be considered were also included in the multivariable model, although they did not reach a $P$-value $<0.25$. A $P$-value $<0.05$ was used to declare the level of statistical significance in the multivariable analysis, and adjusted prevalence ratios (APRs) along with 95\% CIs were estimated. The log-likelihood ratio test and Akaike's and Bayesian information criteria were used to select the final model. The Pearson chi-squared and HosmerLemeshow goodness-of-fit tests were used to test for model fitness. The explanatory variables were tested for multi-collinearity before entering them into multivariable models using variance inflation factor values of less than 10 .

\section{Results}

\section{Characteristics of Study Participants}

A total of 3015 postpartum women were included in the study. The majority of the participating women were formally married to their partner $(2922,96.9 \%)$. The majority 
of them were Muslims (88.4\%), had no formal education $(64.0 \%)$ and were housewives (87.9\%). Thre-quarters of the participants were in the middle age group (20-34 years), the overall age range being 15-44 years with mean \pm standard deviation of $26.4 \pm 5.8$ years. A little more than one-quarter (27.3\%) stated that they had a living arrangement together with their own or their partner's parents/families. The participants had nearly equal proportions in their household empowerment status of low, medium and high levels. Few of the women $(0.7 \%)$ or their husbands/partners $(3.3 \%)$ were frequent alcohol drinkers during the pregnancy period. The majority of the women's husbands/partners $(84.6 \%)$ were habitual khat chewers. Nearly half (45.8\%) of the participants' husbands/partners had no formal education and $54.8 \%$ of them encouraged the women's ANC use. Half of the women who participated in the study $(50.1 \%)$ stated that they had not attended antenatal care (ANC). The majority of the studied women and their husbands/partners (87.9\%) had concordant/agreed intentions in the index pregnancy (Table 1).

\section{Prevalence of Intimate Partner Violence During Pregnancy}

Among the 3015 study participants, 918 experienced at least one type of violent act during pregnancy (any or overall IPVP), corresponding to a prevalence of $30.5 \%$ (95\% CI: 28.8, 32.1). Psychological violence was the most common form, with a prevalence of $24.4 \%$ (95\% CI: $22.9,26.0)$, followed by physical $(11.9 \%$; $95 \% \mathrm{CI}$ : $10.8,13.2)$ and sexual violence $(11.0 \% ; 95 \% \mathrm{CI}$ : $9.9,12.2)$. Of the acts of physical violence, the act of forcefully pushing the pregnant woman was cited as the commonest form of violence, with a prevalence of $7.8 \%$ (95\% CI: 10.8, 13.2). The commonest form of psychological violence was insulting (23.2\%; 95\% CI: 21.7, 24.8), whereas physically forcing the pregnant women to have sexual intercourse was reported as the most frequent act of sexual violence, with a prevalence of $10.4 \%(95 \% \mathrm{CI}$ : $9.3,11.6)$. Of the sexual violence acts, $94.6 \%(314 / 332)$ were in the form of coerced sex. The most frequently overlapping occurrence of IPVP was physical and psychological $(9.3 \%$; 95\% CI: 8.3, 10.4), followed by psychological and sexual $(7.1 \%$; $95 \%$ CI: $6.2,8.1)$ and physical and sexual IPVP $(4.6 \%$; 95\% CI: $3.9,5.4)$. The joint occurrence of all types of IPVP was $4.0 \%$ (95\% CI: $3.3,4.8)$ (Table 2).

\section{Factors Associated with Intimate Partner Violence During Pregnancy}

In the results of bivariate analysis, all forms of IPVP (physical, sexual, psychological and any/overall IPVP) were significantly more prevalent among non-educated women and their husband/partner. On the other hand, the experience of all forms of IPVP was shown to significantly decrease as women advanced in education from primary or secondary to tertiary level. All forms of IPVP tended to be more prevalent among working women and among those with medium to high levels of household empowerment (Tables 3-6).

The bivariate analysis also shows that the prevalence of psychological, physical and any IPVP significantly increased as the women's age advanced (reaching a peak among the age group 35 years and above) and the number of living children increased (reaching a peak among women with seven or more children). Specifically, the CPRs for women aged 35 years and above for psychological, physical and any IPVP were 2.00 (1.46, 2.73; Table 3), 2.15 (1.33, 3.49; Table 4) and 1.49 (1.16, 1.91; Table 6), respectively. The CPRs for women with seven or more children for psychological, physical and any-IPVP were 1.42 (1.16, 1.73; Table 3), 1.59 (1.18, 2.15; Table 4) and 1.19 (1.01, 1.43; Table 6), respectively. Moreover, in this bivariate analysis significant associations were detected between husband's/partner's education, women's working status and the couple's pregnancy intentions with each of the violence types; ie psychological IPVP (Table 3), Physical IPVP (Table 4), sexual IPVP (Table 5) and overall IPVP (Table 6).

In a multivariable analysis, women's education, their working status and having a medium household empowerment level were significantly associated with all forms of IPVP (ie, psychological, physical, sexual and any/overall IPVP). Women who were working were 1.9 times more psychologically abused (APR=1.90; 95\% CI: 1.58, 2.28), 2.54 times more physically abused (APR $=2.54 ; 95 \%$ CI: 1.89 , 3.41), 3.37 times more often victims of sexual IPV $(\mathrm{APR}=3.37 ; 95 \%$ CI: $2.54,4.47)$ and 2.13 times more likely to experience any of these forms of violence during pregnancy ( $\mathrm{APR}=2.13 ; 95 \% \mathrm{CI}: 1.83,2.48)$ compared to housewives. Being educated to tertiary level decreased the PR of psychological abuse by $51 \%$ (APR $=0.49 ; 95 \% \mathrm{CI}: 0.31$, 0.78 ), physical abuse by $95 \%$ ( $\mathrm{APR}=0.05 ; 95 \% \mathrm{CI}: 0.01$, 0.23 ), sexual abuse by $80 \%(\mathrm{APR}=0.20 ; 95 \% \mathrm{CI}: 0.09$, 
Table I Characteristics of Study Participants in Eastern Ethiopia, $2018(n=3015)$

\begin{tabular}{|c|c|c|c|}
\hline Variable & Variable Category & Number & Percent \\
\hline Residence & $\begin{array}{l}\text { Harar } \\
\text { Kersa }\end{array}$ & $\begin{array}{l}542 \\
2473\end{array}$ & $\begin{array}{l}18.0 \\
82.0\end{array}$ \\
\hline Religion & $\begin{array}{l}\text { Muslim } \\
\text { Orthodox } \\
\text { Protestant }\end{array}$ & $\begin{array}{l}2666 \\
296 \\
53\end{array}$ & $\begin{array}{l}88.4 \\
9.8 \\
1.8\end{array}$ \\
\hline Age group (years) & $\begin{array}{l}<20 \\
20-34 \\
\geq 35\end{array}$ & $\begin{array}{l}333 \\
2284 \\
398\end{array}$ & $\begin{array}{l}11.0 \\
75.8 \\
13.2\end{array}$ \\
\hline Age at first marriage (years) & $\begin{array}{l}<18 \\
\geq 18\end{array}$ & $\begin{array}{l}1596 \\
1419\end{array}$ & $\begin{array}{l}52.9 \\
47.1\end{array}$ \\
\hline Marital duration (years) & $\begin{array}{l}\leq 1 \\
2-4 \\
5-10 \\
>10\end{array}$ & $\begin{array}{l}303 \\
618 \\
1111 \\
983\end{array}$ & $\begin{array}{l}10.1 \\
20.5 \\
36.8 \\
32.6\end{array}$ \\
\hline Education (women) & $\begin{array}{l}\text { No education } \\
\text { Primary or secondary } \\
\text { Tertiary }\end{array}$ & $\begin{array}{l}1932 \\
927 \\
156\end{array}$ & $\begin{array}{l}64.1 \\
30.8 \\
5.1\end{array}$ \\
\hline Education (partner) & $\begin{array}{l}\text { No education } \\
\text { Primary or secondary } \\
\text { Tertiary }\end{array}$ & $\begin{array}{l}1382 \\
1388 \\
245\end{array}$ & $\begin{array}{l}45.8 \\
46.0 \\
8.2\end{array}$ \\
\hline Women's household empowerment status & $\begin{array}{l}\text { Low } \\
\text { Medium } \\
\text { High }\end{array}$ & $\begin{array}{l}1006 \\
1006 \\
1003\end{array}$ & $\begin{array}{l}33.4 \\
33.4 \\
33.2\end{array}$ \\
\hline Drinking habit (women) & $\begin{array}{l}\text { Yes, frequently } \\
\text { Yes, occasionally } \\
\text { Not at all }\end{array}$ & $\begin{array}{l}22 \\
158 \\
283 \mid\end{array}$ & $\begin{array}{l}0.7 \\
5.3 \\
94.0\end{array}$ \\
\hline Drinking habit (partner) & $\begin{array}{l}\text { Yes, frequently } \\
\text { Yes, occasionally } \\
\text { Not at all } \\
\text { Do not know }\end{array}$ & $\begin{array}{l}98 \\
272 \\
2547 \\
77\end{array}$ & $\begin{array}{l}3.3 \\
9.1 \\
85.1 \\
2.5\end{array}$ \\
\hline Khat-chewing habit (partner) & $\begin{array}{l}\text { Never/not habitually } \\
\text { Always/habitually }\end{array}$ & $\begin{array}{l}464 \\
2551\end{array}$ & $\begin{array}{l}15.4 \\
84.6\end{array}$ \\
\hline Living arrangement & $\begin{array}{l}\text { Not with family } \\
\text { With family }\end{array}$ & $\begin{array}{l}2192 \\
823\end{array}$ & $\begin{array}{l}72.7 \\
27.3\end{array}$ \\
\hline Working status (women) & $\begin{array}{l}\text { Housewife } \\
\text { Working }\end{array}$ & $\begin{array}{l}2650 \\
365\end{array}$ & $\begin{array}{l}87.9 \\
12.1\end{array}$ \\
\hline Working status (partner) & $\begin{array}{l}\text { Working } \\
\text { Not working }\end{array}$ & $\begin{array}{l}2940 \\
75\end{array}$ & $\begin{array}{l}97.5 \\
2.5\end{array}$ \\
\hline Number of children under 5 at home & $\begin{array}{l}<3 \\
\geq 3\end{array}$ & $\begin{array}{l}2647 \\
368\end{array}$ & $\begin{array}{l}87.8 \\
12.2\end{array}$ \\
\hline ANC attendance & $\begin{array}{l}\text { Not attended } \\
\text { Attended } \geq 1\end{array}$ & $\begin{array}{l}1511 \\
1504\end{array}$ & $\begin{array}{l}50.1 \\
49.9\end{array}$ \\
\hline
\end{tabular}

(Continued) 
Table I (Continued).

\begin{tabular}{|l|l|l|l|}
\hline Variable & Variable Category & Number & Percent \\
\hline Partner's stance on ANC use & Discouraged & 56 & 1.9 \\
& No interest & 1305 & 43.3 \\
& Encouraged & 1654 & 54.8 \\
\hline Number of living children & $\leq 3$ & 1867 & 61.9 \\
& $4-6$ & 878 & 29.1 \\
\hline Couple's pregnancy intentions & $\geq 7$ & 270 & 9.0 \\
\hline Previous infant death & Concordant & 2649 & 87.9 \\
& Discordant & 366 & 12.1 \\
\hline
\end{tabular}

Abbreviation: ANC, antenatal care.

Table 2 Prevalence of Different Types of Intimate Partner Violence During Pregnancy, Eastern Ethiopia, 2018 ( $n=3015)$

\begin{tabular}{|c|c|c|}
\hline Violent Act/Item & Number & Prevalence, \% (95\% Cl) \\
\hline Physical (at least one of the six below) & 361 & $11.9(10.8,13.2)$ \\
\hline Being pushed & 234 & $7.8(6.8,8.8)$ \\
\hline Being slapped & 184 & $6.1(5.3,7.0)$ \\
\hline Being punched with fist & 115 & $3.8(3.2,4.6)$ \\
\hline Being kicked or dragged & 23 & $0.8(0.5,1.1)$ \\
\hline Being choked or burnt intentionally & 11 & $0.4(0.2,0.7)$ \\
\hline Being threatened or actually attacked with weapon $/ \mathrm{knife}$ & 17 & $0.6(0.3,0.9)$ \\
\hline Psychological (at least one of the four below) & 737 & $24.4(22.9,26.0)$ \\
\hline Being insulted & 700 & $23.2(21.7,24.8)$ \\
\hline Being belittled/humiliated & 294 & $9.8(8.7,10.9)$ \\
\hline Being scared/intimidated on purpose & 85 & $2.8(2.3,3.5)$ \\
\hline Being threatened to hurt her or someone she cares about & 30 & $1.0(0.7,1.4)$ \\
\hline Sexual (at least one of the three below) & 332 & $11.0(9.9,12.2)$ \\
\hline Being physically forced to have sex & 314 & $10.4(9.3,11.6)$ \\
\hline Had sex when not wanting to because of fear & 116 & $3.9(3.2,4.6)$ \\
\hline Forced to do something sexual which is humiliating & 35 & $1.2(0.8,1.6)$ \\
\hline Any (overall) IPVP ${ }^{\dagger}$ & 918 & $30.5(28.8,32.1)$ \\
\hline \multicolumn{3}{|l|}{ Overlapping Occurrences* } \\
\hline Physical and Psychological & 281 & $9.3(8.3,10.4)$ \\
\hline Physical and Sexual & 138 & $4.6(3.9,5.4)$ \\
\hline Psychological and Sexual & 214 & $7.1(6.2,8.1)$ \\
\hline Physical, Psychological and Sexual & 121 & $4.0(3.3,4.8)$ \\
\hline
\end{tabular}

Notes: *Joint occurrences of physical, psychological and sexual violence during pregnancy. ${ }^{\dagger}$ At least one act of the 13 acts of violence comprising physical, sexual and psychological violence.

$0.45)$ and overall abuse by $64 \%(\mathrm{APR}=0.36 ; 95 \% \mathrm{CI}: 0.24$, $0.53)$ compared to the non-educated women. Women with a medium household empowerment level showed a significant and positive association in the prevalence of all forms of IPVP compared to women with a low level of empowerment (Table 7).
Women's alcohol drinking habit during pregnancy and their experience of infant loss were significantly associated with all forms of IPVP, except for the physical type. Women who had no habit of drinking alcohol experienced 47\% less psychological IPVP (APR $=0.53$; 95\% CI: 0.29 , 0.99), 75\% less sexual IPVP (APR=0.25; 95\% CI: 0.12, 
Table 3 Bivariate Associations Between Various Factors and Psychological Intimate Partner Violence During Pregnancy, Eastern Ethiopia $(n=3015), 2018$

\begin{tabular}{|c|c|c|c|c|c|}
\hline \multirow[t]{2}{*}{ Variable } & \multirow[t]{2}{*}{ Category } & \multicolumn{2}{|c|}{ Psychological IPVP } & \multirow[t]{2}{*}{ CPR $(95 \% \mathrm{Cl})$} & \multirow[t]{2}{*}{ P-value } \\
\hline & & Yes, n (\%) & No, $n(\%)$ & & \\
\hline \multirow[t]{2}{*}{ Residence } & Harar & $135(24.9)$ & $407(75.1)$ & 1.00 & \\
\hline & Kersa & $602(24.3)$ & |87|(75.7) & $0.98(0.83,1.15)$ & 0.781 \\
\hline \multirow[t]{3}{*}{ Age group(years) } & $<20$ & $46(13.8)$ & $287(86.2)$ & 1.00 & \\
\hline & $20-34$ & $581(25.4)$ & $1703(74.6)$ & $1.84(1.39,2.43)$ & 0.000 \\
\hline & $\geq 35$ & $110(27.6)$ & $288(72.4)$ & $2.00(1.46,2.73)$ & 0.000 \\
\hline \multirow[t]{2}{*}{ Age at first marriage (years) } & $<18$ & $404(25.3)$ & $1192(74.7)$ & 1.00 & \\
\hline & $\geq 18$ & $333(23.5)$ & $1086(76.5)$ & $0.9(0.81,1.05)$ & 0.250 \\
\hline \multirow[t]{4}{*}{ Marital duration (years) } & $\leq 1$ & $57(18.8)$ & $246(81.2)$ & 1.00 & \\
\hline & $2-4$ & $139(22.5)$ & $479(77.5)$ & $1.2(0.90,1.57)$ & 0.204 \\
\hline & $5-10$ & $246(22.1)$ & $865(77.9)$ & I.I $(0.91, \mid .52)$ & 0.217 \\
\hline & $>10$ & $295(30.0)$ & $688(70.0)$ & $1.6(1.23,2.05)$ & 0.000 \\
\hline \multirow[t]{3}{*}{ Education (women) } & No formal education & $519(26.8)$ & $14 \mid 3(73.2)$ & 1.00 & \\
\hline & Primary/secondary & 194(20.9) & 733(79.1) & $0.78(0.67,0.90)$ & 0.000 \\
\hline & Tertiary & $24(15.4)$ & $132(84.6)$ & $0.57(0.39,0.83)$ & 0.004 \\
\hline \multirow[t]{3}{*}{ Education (partner) } & No formal education & $375(27.1)$ & $1007(72.9)$ & 1.00 & \\
\hline & Primary/secondary & $316(22.8)$ & $1072(77.2)$ & $0.84(0.74,0.96)$ & 0.008 \\
\hline & Tertiary & $46(18.8)$ & 199(81.2) & $0.69(0.53,0.91)$ & 0.009 \\
\hline \multirow[t]{3}{*}{ Household empowerment status } & Low & $202(20.1)$ & $804(79.9)$ & 1.00 & \\
\hline & Medium & $316(31.4)$ & $690(68.6)$ & $1.56(1.34,1.82)$ & 0.000 \\
\hline & High & $219(21.8)$ & $784(78.2)$ & $1.09(0.92,1.29)$ & 0.334 \\
\hline \multirow[t]{3}{*}{ Drinking habit (women) } & Yes frequently & $7(31.8)$ & $15(68.2)$ & 1.00 & \\
\hline & Yes occasionally & $5 \mathrm{I}(32.3)$ & $107(67.7)$ & $1.01(0.53,1.95)$ & 0.966 \\
\hline & Not at all & $677(23.9)$ & $2154(76.1)$ & $0.75(0.41,1.39)$ & 0.363 \\
\hline \multirow[t]{3}{*}{ Drinking habit (partner) } & Yes frequently & $27(27.6)$ & $7 I(72.4)$ & 1.00 & \\
\hline & Yes occasionally & $68(25.0)$ & $204(75.0)$ & $0.91(0.62,1.33)$ & 0.618 \\
\hline & Not at all & $600(23.6)$ & $1947(76.4)$ & $0.85(0.62,1.19)$ & 0.350 \\
\hline \multirow[t]{2}{*}{ Khat-chewing habit (partner) } & Never/occasional & $77(16.6)$ & $387(83.4)$ & 1.00 & \\
\hline & Frequently & $660(25.9)$ & $189 \mid(74.1)$ & $1.56(1.26,1.93)$ & 0.000 \\
\hline \multirow[t]{2}{*}{ Working status (women) } & Housewife & $598(22.6)$ & $2052(77.4)$ & 1.00 & \\
\hline & Working & $139(38.1)$ & $226(61.9)$ & $1.7(1.45,1.95)$ & 0.000 \\
\hline \multirow[t]{2}{*}{ Working status (partner) } & Working & $716(24.4)$ & $2224(75.6)$ & 1.00 & \\
\hline & Non-working & $21(28.0)$ & $54(72.0)$ & $1.15(0.80,1.66)$ & 0.460 \\
\hline \multirow[t]{2}{*}{ Living arrangement } & Not with family & $538(24.5)$ & $1654(75.5)$ & 1.00 & \\
\hline & With family & $199(24.2)$ & $624(75.8)$ & $0.99(0.86,1.13)$ & 0.836 \\
\hline \multirow[t]{2}{*}{ Number of children under 5} & $<3$ & $646(24.4)$ & $200 I(75.6)$ & 1.00 & \\
\hline & $\geq 3$ & $91(24.7)$ & $277(75.3)$ & I.I $(0.84, I .23)$ & 0.890 \\
\hline \multirow[t]{2}{*}{ ANC attendance } & Not attend & $359(23.8)$ & $1152(76.2)$ & 1.00 & \\
\hline & Attended $\geq 1$ & $378(25.1)$ & $1126(74.9)$ & I.I $(093,1.19)$ & 0.380 \\
\hline \multirow[t]{3}{*}{ Partner's stance on ANC use } & Discourage & $15(26.8)$ & $4 I(73.2)$ & 1.00 & \\
\hline & No interest & $385(29.5)$ & $920(70.5)$ & I.I0(0.7I,I.7I) & 0.668 \\
\hline & Encourage & $337(20.4)$ & $13 \mid 7(79.6)$ & $0.76(0.49,1.18)$ & 0.227 \\
\hline
\end{tabular}


Table 3 (Continued).

\begin{tabular}{|c|c|c|c|c|c|}
\hline \multirow[t]{2}{*}{ Variable } & \multirow[t]{2}{*}{ Category } & \multicolumn{2}{|c|}{ Psychological IPVP } & \multirow[t]{2}{*}{ CPR $(95 \% \mathrm{Cl})$} & \multirow[t]{2}{*}{ P-value } \\
\hline & & Yes, n (\%) & No, n(\%) & & \\
\hline \multirow[t]{3}{*}{ Number of living children } & $\leq 3$ & $404(21.6)$ & I463(78.4) & 1.00 & \\
\hline & $4-6$ & $250(28.5)$ & $628(71.5)$ & $1.32(I .15, \mid .5 I)$ & 0.000 \\
\hline & $\geq 7$ & $83(30.7)$ & $187(69.3)$ & $1.42(1.16,1.73)$ & 0.000 \\
\hline \multirow[t]{2}{*}{ Couple's pregnancy intentions } & Concordant & $622(23.5)$ & $2027(76.5)$ & 1.00 & \\
\hline & Discordant & $115(31.4)$ & $25 \mathrm{I}(68.6)$ & $1.34(1.13,1.58)$ & 0.000 \\
\hline \multirow[t]{2}{*}{ Previous infant loss } & No & $617(24.9)$ & $1858(75.1)$ & 1.00 & \\
\hline & Yes & $120(22.2)$ & $420(77.8)$ & $0.9(0.75,1.05)$ & 0.190 \\
\hline
\end{tabular}

Abbreviations: IPVP, intimate partner violence during pregnancy; CPR, crude prevalence ratio; ANC, antenatal care.

0.57 ) and $45 \%$ less overall IPVP (APR $=0.55 ; 95 \% \mathrm{CI}$ : $0.35,0.87)$ compared to women with a frequent habit of alcohol drinking. Women who experienced infant loss reported a lower prevalence of IPVP of any type, except for physical IPVP (Table 7).

The living arrangements of the couple, woman's age, husband's/partner's khat-chewing habit and the couple's pregnancy intentions were significantly associated with all forms of IPVP, except for the sexual type. Women who were living with their or husband's/partner's family showed a decreased PR for psychological abuse by $19 \%$ (APR $=0.81 ; 95 \%$ CI: $0.69,0.94)$, forphysical abuse by $27 \%(\mathrm{APR}=0.73 ; 95 \% \mathrm{CI}: 0.58,0.94)$ and for any/overall abuse by $13 \%$ (APR $=0.87 ; 95 \%$ CI: $0.77,0.98)$ compared to women living with their husband/partner alone. Older women experienced twice as much physical IPVP, 1.601.83 times higher psychological IPVP and 1.56 times higher any/overall IPVP than younger women, aged less than 20 years. Women whose husband/partner chewed khat frequently and those who had disagreements over the intention of the index pregnancy with their husband/ partner showed a higher prevalence of IPVP across all types, except for sexual IPVP (Table 7).

Women's places of residence were found to be significantly associated with sexual and psychological IPVP. Women residing in rural Kersa had significantly lower rates of sexual (APR $=0.41 ; 95 \% \mathrm{CI}: 0.27,0.60)$ and psychological IPVP (APR $=0.68$; 95\% CI: $0.53,0.87$ ) compared to their counterparts in the urban Harar area. Compared to women whose husband/partner was engaged in work, women with a non-working husband/partner reported a decreased prevalence of sexual and any/overall IPVP (Table 7).
Women whose husband/partner had primary or secondary education were $26 \%$ less abused sexually by their husband/partner than women with a non-educated husband/partner $\quad(\mathrm{APR}=0.74 ; 95 \%$ CI: 0.58, 0.96). Women whose partner encouraged their ANC use also experienced $56 \%$ less physical abuse during pregnancy (APR $=0.44 ; 95 \%$ CI: $0.25,0.78)$ than women who were discouraged from using ANC by their partner (Table 7).

\section{Discussion}

The findings from this study revealed that the prevalences of psychological, physical and sexual IPVP were $24.4 \%$, $11.9 \%$ and $11.0 \%$, respectively. This order of prevalence of IPVP is consistent with study reports from Ethiopia ${ }^{14}$ and elsewhere. ${ }^{46-48}$ The finding that psychological violence was the most prevalent abusive act in this study may be due to the fact that pregnancy could offer protection against physical violence for many women, and that the decrease in physical violence during the pregnancy period may parallel an increase in psychological violence, as evidenced by the patterns of longitudinal violence prevalence in relation to pregnancy onset in studies from Brazil $^{49}$ and Bangladesh. ${ }^{9}$ Moreover, in this study, the use of the culturally sensitive WHO Violence Against Women questionnaire is considered to increase detection of the emotionally abusive acts of insulting, intimidating and threatening, which might not be culturally considered as acts of violence in many traditional societies. Notably, this study also indicated that the most common co-occurring types of violence during pregnancy were psychological and physical violence. Comparable IPVP overlap has been reported in previous studies. ${ }^{9,49}$ 
Table 4 Bivariate Associations Between Various Factors and Physical Intimate Partner Violence During Pregnancy, Eastern Ethiopia $(n=3015), 2018$

\begin{tabular}{|c|c|c|c|c|c|}
\hline \multirow[t]{2}{*}{ Variable } & \multirow[t]{2}{*}{ Category } & \multicolumn{2}{|c|}{ Physical IPVP } & \multirow[t]{2}{*}{ CPR $(95 \% \mathrm{Cl})$} & \multirow[t]{2}{*}{ P-value } \\
\hline & & Yes, $n(\%)$ & No, $n(\%)$ & & \\
\hline Residence & $\begin{array}{l}\text { Harar } \\
\text { Kersa }\end{array}$ & $\begin{array}{l}50(9.2) \\
311(12.6)\end{array}$ & $\begin{array}{l}492(90.8) \\
2162(87.4)\end{array}$ & $\begin{array}{l}1.00 \\
1.36(1.03,1.81)\end{array}$ & 0.032 \\
\hline Age group(years) & $\begin{array}{l}<20 \\
20-34 \\
\geq 35\end{array}$ & $\begin{array}{l}2 I(6.3) \\
286(12.5) \\
54(13.6)\end{array}$ & $\begin{array}{l}312(93.7) \\
1998(87.5) \\
344(86.4)\end{array}$ & $\begin{array}{l}1.00 \\
1.98(1.29,3.05) \\
2.15(1.33,3.49)\end{array}$ & $\begin{array}{l}0.002 \\
0.002\end{array}$ \\
\hline Age at first marriage (years) & $\begin{array}{l}<18 \\
\geq 18\end{array}$ & $\begin{array}{l}209(13.1) \\
152(10.7)\end{array}$ & $\begin{array}{l}1387(86.9) \\
1267(89.3)\end{array}$ & $\begin{array}{l}1.00 \\
0.8(0.67,0.99)\end{array}$ & 0.045 \\
\hline Marital duration (years) & $\begin{array}{l}\leq 1 \\
2-4 \\
5-10 \\
>10\end{array}$ & $\begin{array}{l}23(7.6) \\
69(\mid 1.2) \\
123(\mid 1.1) \\
146(14.9)\end{array}$ & $\begin{array}{l}280(92.4) \\
549(88.8) \\
988(88.9) \\
837(85.1)\end{array}$ & $\begin{array}{l}1.00 \\
1.5(0.94,2.31) \\
1.4(0.95,2.23) \\
1.9(1.28,2.97)\end{array}$ & $\begin{array}{l}0.094 \\
0.083 \\
0.002\end{array}$ \\
\hline Education (women) & $\begin{array}{l}\text { No formal education } \\
\text { Primary/secondary } \\
\text { Tertiary }\end{array}$ & $\begin{array}{l}283(14.7) \\
76(8.2) \\
2(1.3)\end{array}$ & $\begin{array}{l}1649(85.3) \\
851(91.8) \\
154(98.7)\end{array}$ & $\begin{array}{l}1.00 \\
0.56(0.44,0.71) \\
0.09(0.02,0.35)\end{array}$ & $\begin{array}{l}0.000 \\
0.001\end{array}$ \\
\hline Education (partner) & $\begin{array}{l}\text { No formal education } \\
\text { Primary/secondary } \\
\text { Tertiary }\end{array}$ & $\begin{array}{l}204(14.8) \\
142(10.2) \\
15(6.1)\end{array}$ & $\begin{array}{l}1178(85.2) \\
1246(89.8) \\
230(93.9)\end{array}$ & $\begin{array}{l}1.00 \\
0.69(0.57,0.85) \\
0.41(0.25,0.69)\end{array}$ & $\begin{array}{l}0.000 \\
0.001\end{array}$ \\
\hline Household empowerment status & $\begin{array}{l}\text { Low } \\
\text { Medium } \\
\text { High }\end{array}$ & $\begin{array}{l}107(10.6) \\
166(16.5) \\
88(8.8)\end{array}$ & $\begin{array}{l}899(89.4) \\
840(83.5) \\
915(91.2)\end{array}$ & $\begin{array}{l}1.00 \\
1.55(1.23,1.95) \\
0.82(0.63,1.08)\end{array}$ & $\begin{array}{l}0.000 \\
0.159\end{array}$ \\
\hline Drinking habit (women) & $\begin{array}{l}\text { Yes frequently } \\
\text { Yes occasionally } \\
\text { Not at all }\end{array}$ & $\begin{array}{l}4(18.2) \\
22(13.9) \\
335(11.8)\end{array}$ & $\begin{array}{l}18(81.8) \\
136(86.1) \\
2496(88.2)\end{array}$ & $\begin{array}{l}1.00 \\
0.77(0.29,2.02) \\
0.65(0.27,1.59)\end{array}$ & $\begin{array}{l}0.589 \\
0.345\end{array}$ \\
\hline Drinking habit (partner) & $\begin{array}{l}\text { Yes frequently } \\
\text { Yes occasionally } \\
\text { Not at all }\end{array}$ & $\begin{array}{l}9(9.2) \\
30(11.0) \\
287(11.3)\end{array}$ & $\begin{array}{l}89(90.8) \\
242(89.0) \\
2260(88.7)\end{array}$ & $\begin{array}{l}1.00 \\
1.20(0.59,2.44) \\
1.23(0.65,2.31)\end{array}$ & $\begin{array}{l}0.612 \\
0.526\end{array}$ \\
\hline Khat-chewing habit (partner) & $\begin{array}{l}\text { Never/occasional } \\
\text { Frequently }\end{array}$ & $\begin{array}{l}22(4.7) \\
339(13.3)\end{array}$ & $\begin{array}{l}442(95.3) \\
2212(86.7)\end{array}$ & $\begin{array}{l}1.00 \\
2.80(1.84,4.26)\end{array}$ & 0.000 \\
\hline Working status (women) & $\begin{array}{l}\text { Housewife } \\
\text { Working }\end{array}$ & $\begin{array}{l}292(11.0) \\
69(18.9)\end{array}$ & $\begin{array}{l}2358(89.0) \\
296(81.1)\end{array}$ & $\begin{array}{l}1.00 \\
1.7(1.35,2.18)\end{array}$ & 0.000 \\
\hline Working status (partner) & $\begin{array}{l}\text { Working } \\
\text { Non-working }\end{array}$ & $\begin{array}{l}355(12.1) \\
6(8.0)\end{array}$ & $\begin{array}{l}2585(87.9) \\
69(92.0)\end{array}$ & $\begin{array}{l}1.00 \\
0.66(0.31,1.44)\end{array}$ & 0.297 \\
\hline Living arrangement & $\begin{array}{l}\text { Not with family } \\
\text { With family }\end{array}$ & $\begin{array}{l}259(11.8) \\
102(12.4)\end{array}$ & $\begin{array}{l}1933(88.2) \\
721(87.6)\end{array}$ & $\begin{array}{l}1.00 \\
1.05(0.85,1.30)\end{array}$ & 0.663 \\
\hline Number of children under 5 & $\begin{array}{l}<3 \\
\geq 3\end{array}$ & $\begin{array}{l}308(11.6) \\
53(14.4)\end{array}$ & $\begin{array}{l}2339(88.4) \\
315(85.6)\end{array}$ & $\begin{array}{l}1.00 \\
1.2(094,1.62)\end{array}$ & 0.122 \\
\hline ANC attendance & $\begin{array}{l}\text { Not attend } \\
\text { Attended } \geq 1\end{array}$ & $\begin{array}{l}179(11.9) \\
182(12.1)\end{array}$ & $\begin{array}{l}1332(88.1) \\
1322(87.9)\end{array}$ & $\begin{array}{l}\text { I.00 } \\
\text { I.I }(0.84,1.24)\end{array}$ & 0.830 \\
\hline Partner's stance on ANC use & $\begin{array}{l}\text { Discourage } \\
\text { No interest } \\
\text { Encourage }\end{array}$ & $\begin{array}{l}10(17.9) \\
182(13.9) \\
169(10.2)\end{array}$ & $\begin{array}{l}46(82.1) \\
1123(86.1) \\
1485(89.8)\end{array}$ & $\begin{array}{l}1.00 \\
0.78(0.44,1.39) \\
0.57(0.32,1.02)\end{array}$ & $\begin{array}{l}0.402 \\
0.059\end{array}$ \\
\hline
\end{tabular}


Table 4 (Continued).

\begin{tabular}{|c|c|c|c|c|c|}
\hline \multirow[t]{2}{*}{ Variable } & \multirow[t]{2}{*}{ Category } & \multicolumn{2}{|c|}{ Physical IPVP } & \multirow[t]{2}{*}{ CPR $(95 \% \mathrm{Cl})$} & \multirow[t]{2}{*}{ P-value } \\
\hline & & Yes, n(\%) & No, $n(\%)$ & & \\
\hline \multirow[t]{3}{*}{ Number of living children } & $\leq 3$ & $191(10.2)$ & $1676(89.8)$ & 1.00 & \\
\hline & 4-6 & $126(14.4)$ & $752(85.6)$ & $1.40(1.14,1.73)$ & 0.002 \\
\hline & $\geq 7$ & $44(16.3)$ & $226(83.7)$ & $1.59(1.18,2.15)$ & 0.003 \\
\hline \multirow[t]{2}{*}{ Couple's pregnancy intentions } & Concordant & $301(11.4)$ & $2348(88.6)$ & 1.00 & \\
\hline & Discordant & $60(16.4)$ & $306(83.6)$ & $1.44(1.11,1.86)$ & 0.005 \\
\hline \multirow[t]{2}{*}{ Previous infant loss } & No & $301(12.2)$ & $2174(87.8)$ & 1.00 & \\
\hline & Yes & $60(11.1)$ & $480(88.9)$ & $0.9(0.70,1.19)$ & 0.498 \\
\hline
\end{tabular}

Abbreviations: IPVP, intimate partner violence during pregnancy; CPR, crude prevalence ratio; ANC, antenatal care.

In this study, the overall prevalence of IPVP was $30.5 \%$ (95\% CI: 28.8, 32.1). This result is comparable to that found by Yimer et al in Northwest Ethiopia, ${ }^{31}$ indicating a prevalence rate of $32.2 \%(95 \% \mathrm{CI}: 27.8,36.5)$, and to the finding of a 2018 review on IPVP in Ethiopia that demonstrated pooled prevalence estimates of $26.1 \%(95 \%$ CI: $20.0,32.3) .{ }^{14}$ Other community-based studies conducted in Ethiopia ${ }^{29}$ and Bangladesh, ${ }^{50}$ however, reported a much higher rate of overall IPVP than the findings of this study. This may be because our sample comprised mothers who had given birth very recently (within 6 weeks of delivery), and the excitement of the birth may have led to a lower likelihood of reporting IPV experienced during the index pregnancy.

This study revealed that $94.6 \%$ of the sexual violence acts during pregnancy were in the form of coerced sex. This proportion is far greater than in the studies conducted by Yimer et $\mathrm{al}^{31}$ and Abate et al, ${ }^{29}$ where the sexually coercive act was reported at $66.7 \%$ and $65.8 \%$, respectively. In Ethiopia, the high prevalence of marital forced sex is largely accounted for by the culturally imposed marital sexual relationship, where there is little or no consideration for the woman's consent to sex and it takes place whenever the man wants it. ${ }^{51}$ Sexual violence in the form of forceful sex has severe consequences for the abused pregnant woman as it may lead to gynecological problems including severe injuries, vaginal bleeding or infections. ${ }^{52}$ Such maternal complications are known to lead to pregnancy loss or premature birth, particularly if the forced sex is perpetrated during late pregnancy. ${ }^{53}$ The consequences of IPV and forced sex during pregnancy on adverse birth outcomes were reported in a 2019 study in Ethiopia. ${ }^{54}$

Women who resided in the rural study area (Kersa district) had a significantly lower prevalence of sexual
IPVP than their urban counterparts (women in Harar). This may be explained by underreporting of sexual abuse in the rural women (compared to urban women) due to the sensitivity of the issue and the surrounding cultural taboos, ${ }^{55}$ although several measures such as securing confidentiality and involving female data collectors were in place in this study. The study also revealed that the rural women experienced less psychological IPVP than the urban women, which may indicate that in rural settings, in contrast to urban areas, acts of psychological violence such as insulting and belittling may not be considered as violence, leading to underreporting of these acts.

Being of an older age was significantly and positively associated with all forms of IPVP, except for sexual violence, which agrees with the findings of previous studies. ${ }^{56,57}$ One explanation for this may be older women reporting cumulatively persisting experiences of partner violence, although the validity of this association needs to be treated cautiously as this study is cross-sectional and asserting a cumulative effect may be difficult. However, other studies reported a reverse association, where middle or older aged women reported less experience of IPVP. ${ }^{31,48}$

In this study, education of women provided a protective effect against the occurrence of all forms of IPVP, which agrees with studies conducted in low-resource settings. ${ }^{47,58,59}$ This could be attributed to the fact that education can provide women with ample information on their rights and greater negotiating ability with their husband/partner, which helps in changing patriarchal norms and values. ${ }^{55}$

Compared to women living in separate arrangements, women who were living with families (either their own or their husband's/partner's family) had a significantly lower experience of all forms of violence, except for sexual, 
Table 5 Bivariate Associations Between Various Factors and Sexual Intimate Partner Violence During Pregnancy, Eastern Ethiopia $(n=3015), 2018$

\begin{tabular}{|c|c|c|c|c|c|}
\hline \multirow[t]{2}{*}{ Variable } & \multirow[t]{2}{*}{ Category } & \multicolumn{2}{|c|}{ Sexual IPVP } & \multirow[t]{2}{*}{ CPR $(95 \% \mathrm{Cl})$} & \multirow[t]{2}{*}{ P-value } \\
\hline & & Yes, n (\%) & No, $n(\%)$ & & \\
\hline \multirow[t]{2}{*}{ Residence } & Harar & $84(I 5.5)$ & $458(84.5)$ & 1.00 & \\
\hline & Kersa & $248(10.0)$ & $2225(90.0)$ & $0.65(0.5 I, 0.8 I)$ & 0.000 \\
\hline \multirow{3}{*}{ Age group(years) } & $<20$ & $30(9.0)$ & $303(91.0)$ & 1.00 & \\
\hline & $20-34$ & $25 I(10.9)$ & 2033(89.1) & $1.22(0.85,1.75)$ & 0.280 \\
\hline & $\geq 35$ & $5 I(12.8)$ & $347(87.2)$ & $1.42(0.93,2.18)$ & 0.106 \\
\hline \multirow[t]{2}{*}{ Age at first marriage (years) } & $<18$ & $198(12.4)$ & $1398(87.6)$ & 1.00 & \\
\hline & $\geq 18$ & $134(9.4)$ & $1285(90.6)$ & $0.80(0.62,0.94)$ & 0.010 \\
\hline \multirow[t]{4}{*}{ Marital duration (years) } & $\leq 1$ & $20(6.6)$ & $283(93.4)$ & 1.00 & \\
\hline & $2-4$ & $83(13.4)$ & $535(86.6)$ & $2.1(1.27,3.25)$ & 0.003 \\
\hline & $5-10$ & $96(8.6)$ & $1015(91.4)$ & I.3(0.82,2.08) & 0.256 \\
\hline & $>10$ & $133(13.5)$ & $850(86.5)$ & $2 . I(1.30,3.22)$ & 0.002 \\
\hline \multirow[t]{3}{*}{ Education (women) } & No formal education & $244(12.6)$ & I688(87.4) & 1.00 & \\
\hline & Primary/secondary & $76(8.2)$ & $85 I(91.8)$ & $0.65(0.5 I, 0.83)$ & 0.001 \\
\hline & Tertiary & $12(7.7)$ & I44(92.3) & $0.6 I(0.35,1.06)$ & 0.081 \\
\hline \multirow[t]{3}{*}{ Education (partner) } & No formal education & $188(13.6)$ & $1194(86.4)$ & 1.00 & \\
\hline & Primary/secondary & $117(8.4)$ & $|27|(9 \mid .6)$ & $0.62(0.49,0.77)$ & 0.000 \\
\hline & Tertiary & $27(11.0)$ & $218(89.0)$ & $0.81(0.55,1.18)$ & 0.277 \\
\hline \multirow[t]{3}{*}{ Household empowerment status } & Low & $69(6.9)$ & $937(93.1)$ & 1.00 & \\
\hline & Medium & $183(18.2)$ & $823(81.8)$ & $2.65(2.04,3.45)$ & 0.000 \\
\hline & High & $80(7.9)$ & $923(92.1)$ & $1.16(0.85,1.59)$ & 0.340 \\
\hline \multirow[t]{3}{*}{ Drinking habit (women) } & Yes frequently & $5(22.7)$ & $17(77.3)$ & 1.00 & \\
\hline & Yes occasionally & $36(22.8)$ & $122(77.2)$ & $1.01(0.44,2.28)$ & 0.995 \\
\hline & Not at all & $291(10.3)$ & $2540(89.7)$ & $0.45(0.21,0.98)$ & 0.046 \\
\hline \multirow[t]{3}{*}{ Drinking habit (partner) } & Yes frequently & $10(10.2)$ & $88(89.8)$ & 1.00 & \\
\hline & Yes occasionally & $35(12.9)$ & $237(87.1)$ & $1.26(0.65,2.45)$ & 0.493 \\
\hline & Not at all & $286(I I .2)$ & $2261(88.8)$ & $1.10(0.61,2.99)$ & 0.754 \\
\hline \multirow[t]{2}{*}{ Khat-chewing habit (partner) } & Never/occasional & $53(11.4)$ & $4 \mathrm{II}(88.6)$ & 1.00 & \\
\hline & Frequently & $279(10.9)$ & $2272(89.1)$ & $0.96(0.73,1.26)$ & 0.758 \\
\hline \multirow[t]{2}{*}{ Working status (women) } & Housewife & $208(7.9)$ & $2442(92.1)$ & 1.00 & \\
\hline & Working & $124(34.0)$ & $24 I(66.0)$ & $4.3(3.57,5.25)$ & 0.000 \\
\hline \multirow[t]{2}{*}{ Working status (partner) } & Working & $323(10.9)$ & $2617(89.1)$ & 1.00 & \\
\hline & Non-working & $9(12.0)$ & $66(88.0)$ & $1.09(0.59,2.03)$ & 0.781 \\
\hline \multirow[t]{2}{*}{ Living arrangement } & Not with family & $196(8.9)$ & 1996(91.1) & 1.00 & \\
\hline & With family & $136(16.5)$ & $687(83.5)$ & $1.85(1.51,2.27)$ & 0.000 \\
\hline \multirow[t]{2}{*}{ Number of children under 5} & $<3$ & $295(I I . I)$ & 2352(88.9) & 1.00 & \\
\hline & $\geq 3$ & $37(10.0)$ & $331(90.0)$ & $0.9(0.65,1.25)$ & 0.533 \\
\hline \multirow[t]{2}{*}{ ANC attendance } & Not attend & $116(7.7)$ & $1395(92.3)$ & 1.00 & \\
\hline & Attended $\geq 1$ & $216(\mid 4.4)$ & $1288(85.6)$ & I.8(I.5I,2.32) & 0.000 \\
\hline \multirow[t]{3}{*}{ Partner's stance on ANC use } & Discourage & $\mathrm{I}(\mathrm{I} .8)$ & $55(98.2)$ & 1.00 & \\
\hline & No interest & $159(12.2)$ & $1146(87.8)$ & $6.8(0.97,47.9)$ & 0.053 \\
\hline & Encourage & $172(10.4)$ & | $482(89.6)$ & $5.8(0.83,40.8)$ & 0.076 \\
\hline
\end{tabular}

(Continued) 
Table 5 (Continued).

\begin{tabular}{|c|c|c|c|c|c|}
\hline \multirow[t]{2}{*}{ Variable } & \multirow[t]{2}{*}{ Category } & \multicolumn{2}{|c|}{ Sexual IPVP } & \multirow[t]{2}{*}{ CPR $(95 \% \mathrm{Cl})$} & \multirow[t]{2}{*}{ P-value } \\
\hline & & Yes, n (\%) & No, n(\%) & & \\
\hline \multirow[t]{3}{*}{ Number of living children } & $\leq 3$ & $205(10.9)$ & $1662(89.1)$ & 1.00 & \\
\hline & $4-6$ & $91(10.4)$ & $787(89.6)$ & $0.94(0.75,1.19)$ & 0.628 \\
\hline & $\geq 7$ & $36(13.3)$ & $234(86.7)$ & I.2I $(0.87,1.69)$ & 0.249 \\
\hline \multirow[t]{2}{*}{ Couple's pregnancy intentions } & Concordant & $305(I I .5)$ & $2344(88.5)$ & 1.00 & \\
\hline & Discordant & $27(7.4)$ & $339(92.6)$ & $0.64(0.44,0.94)$ & 0.021 \\
\hline \multirow[t]{2}{*}{ Previous infant loss } & No & $288(I I .6)$ & $2187(88.4)$ & 1.00 & \\
\hline & Yes & $44(8.2)$ & $496(91.8)$ & $0.7(0.52,0.95)$ & 0.021 \\
\hline
\end{tabular}

Abbreviations: IPVP, intimate partner violence during pregnancy; CPR, crude prevalence ratio; ANC, antenatal care.

which is in agreement with a study conducted in a district in Oromia regional state of Ethiopia. ${ }^{29}$ This could be because, if pregnant women are attached to the family and are able to obtain support from them, then their husband's/partner's respect for them may increase and so lower their exposure to violence. ${ }^{60}$

Inconsistent with other studies, ${ }^{47,59,61}$ the findings of this study showed that women who hada medium household empowerment level as well as those working outside the home had a significantly higher experience of all forms of violence from their husband/partner. However, our study findings corroborate study findings from Nigeria. ${ }^{62}$ A possible explanation for this positive association may be that women's confrontation of established male household dominance leads to inconsistencies in male status, thus triggering abuse in order to re-establish power and masculine identity. ${ }^{63-65}$ Besides, many men in Ethiopia culturally believe that women exist for household work and men exist to earn an income and are superior. ${ }^{51}$

The couple's discordant intentions in the index pregnancy were significantly and positively associated with the prevalence of all forms of IPVP, except for sexual. This is in agreement with studies by Pearson et al in Bangladesh ${ }^{66}$ and Shamu et al in Zimbabwe. ${ }^{67}$ It is possible that the pregnancy would trigger an increased risk of violence if it was the result of the woman's lack of reproductive control leading to an unintended pregnancy. ${ }^{68}$ The woman's own decision to become pregnant (without the consent of her husband/partner) may also put her at greater risk of violence, as her decision may be in conflict with her husband's/partner's role as provider in a situation of limited economic resources. ${ }^{67}$

In this study, frequent consumption of alcohol by pregnant women was positively associated with IPVP perpetration by the husband/partner against them. This finding is in agreement with other studies in Ethiopia and other places. ${ }^{31,58,69}$ It may, however, be possible that the pregnant women self-medicated with alcohol as a coping strategy to alleviate the stress and trauma associated with the experience of violence. ${ }^{6,70}$ This bidirectional association warrants further investigation into whether women's consumption of alcohol in pregnancy is more likely to lead to IPVP or whether exposure to IPVP increases alcohol intake in pregnancy. ${ }^{71}$ In this study, frequent khat chewing by the husband/partner contributed to increased exposure of pregnant women to IPVP, which may be due to the negative influence of irresponsible behavior due to the khat addiction. ${ }^{34}$

Inconsistent with prior studies, ${ }^{58,69}$ previous experience of infant death was negatively associated with all forms of IPVP. This may be due to cultural reasons related to the husband's/partner's sympathetic and emotionally supportive attitude as a result of the family's loss of an infant. ${ }^{6,72}$

\section{Strengths and Limitations of the Study}

This study has several strengths. The consideration of all forms of violence (psychological, physical, sexual and overall IPVP) gives us wider scope to observe which factors were highly associated with which of these IPVP types. Moreover, our assessment of IPVP from postpartum mothers whose pregnancies had ended very recently could reduce the level of recall bias in the study. The use of PRs estimated by the log-binomial regression model, which is an appropriate statistical method for cross-sectional studies of high outcome prevalence (usually $>10 \%$ ), is another strength of this study. The narrow confidence intervals observed in the results of this study owing to the study's large sample size also show a good precision in the estimated associations of outcome and factor variables. 
Table 6 Bivariate Associations Between Various Factors and Overall Intimate Partner Violence During Pregnancy, Eastern Ethiopia $(n=3015), 2018$

\begin{tabular}{|c|c|c|c|c|c|}
\hline \multirow[t]{2}{*}{ Variable } & \multirow[t]{2}{*}{ Category } & \multicolumn{2}{|c|}{ Overall IPVP } & \multirow[t]{2}{*}{ CPR $(95 \% \mathrm{Cl})$} & \multirow[t]{2}{*}{ P-value } \\
\hline & & Yes, n (\%) & No, $n(\%)$ & & \\
\hline \multirow[t]{2}{*}{ Residence } & Harar & $176(32.5)$ & $366(67.5)$ & 1.00 & \\
\hline & Kersa & $742(30.0)$ & $|73|(70.0)$ & $0.92(0.81,1.06)$ & 0.253 \\
\hline \multirow[t]{3}{*}{ Age group(years) } & $<20$ & $72(21.6)$ & $26 \mid(78.4)$ & 1.00 & \\
\hline & $20-34$ & $718(31.4)$ & $1566(68.6)$ & $1.45(1.17,1.80)$ & 0.001 \\
\hline & $\geq 35$ & $128(32.2)$ & $270(67.8)$ & $1.49(1.16,1.91)$ & 0.002 \\
\hline \multirow[t]{2}{*}{ Age at first marriage (years) } & $<18$ & $517(32.4)$ & $1079(67.6)$ & 1.00 & \\
\hline & $\geq 18$ & $40 \mathrm{I}(28.3)$ & $1018(71.7)$ & $0.9(0.78,0.97)$ & 0.014 \\
\hline \multirow[t]{4}{*}{ Marital duration (years) } & $\leq 1$ & $75(24.8)$ & $228(75.2)$ & 1.00 & \\
\hline & $2-4$ & $188(30.4)$ & $430(69.6)$ & $1.2(0.98,1.55)$ & 0.078 \\
\hline & $5-10$ & $310(27.9)$ & $80 I(72.1)$ & I.I $(0.91, \mid .40)$ & 0.281 \\
\hline & $>10$ & $345(35.1)$ & $638(64.9)$ & $1.4(1.15,1.76)$ & 0.001 \\
\hline \multirow[t]{3}{*}{ Education (women) } & No formal education & 644(33.3) & $1288(66.7)$ & 1.00 & \\
\hline & Primary/secondary & $244(26.3)$ & 683(73.7) & $0.79(0.69,0.89)$ & 0.000 \\
\hline & Tertiary & $30(19.2)$ & $126(80.8)$ & $0.58(0.42,0.80)$ & 0.001 \\
\hline \multirow[t]{3}{*}{ Education (partner) } & No formal education & $457(33.1)$ & $925(66.9)$ & 1.00 & \\
\hline & Primary/secondary & $397(28.6)$ & $991(71.4)$ & $0.86(0.77,0.97)$ & 0.011 \\
\hline & Tertiary & $64(26.1)$ & I8I(73.9) & $0.79(0.63,0.99)$ & 0.039 \\
\hline \multirow[t]{3}{*}{ Household empowerment status } & Low & $263(26.1)$ & $743(73.9)$ & 1.00 & \\
\hline & Medium & $382(37.9)$ & $624(62.1)$ & $1.45(1.27,1.66)$ & 0.000 \\
\hline & High & $273(27.2)$ & $730(72.8)$ & $1.04(0.90,1.20)$ & 0.586 \\
\hline \multirow[t]{3}{*}{ Drinking habit (women) } & Yes frequently & $9(40.9)$ & 13(59.1) & 1.00 & \\
\hline & Yes occasionally & $70(44.3)$ & $88(55.7)$ & $1.08(0.64,1.84)$ & 0.769 \\
\hline & Not at all & $837(29.6)$ & $1994(70.4)$ & $0.72(0.44,1.20)$ & 0.208 \\
\hline \multirow[t]{3}{*}{ Drinking habit (partner) } & Yes frequently & $32(32.7)$ & $66(67.3)$ & 1.00 & \\
\hline & Yes occasionally & $89(32.7)$ & $183(67.3)$ & $1.01(0.72, I .40)$ & 0.990 \\
\hline & Not at all & $746(29.3)$ & $|80|(70.7)$ & $0.90(0.67,1.20)$ & 0.464 \\
\hline \multirow[t]{2}{*}{ Khat-chewing habit (partner) } & Never/occasional & $110(23.7)$ & $354(76.3)$ & 1.00 & \\
\hline & Frequently & $808(31.7)$ & $1743(68.3)$ & $1.34(1.12,1.58)$ & 0.001 \\
\hline \multirow[t]{2}{*}{ Working status (women) } & Housewife & $734(27.7)$ & $1916(72.3)$ & 1.00 & \\
\hline & Working & $184(50.4)$ & $|8|(49.6)$ & $1.8(1.62,2.05)$ & 0.000 \\
\hline \multirow[t]{2}{*}{ Working status (partner) } & Working & $891(30.3)$ & $2049(69.7)$ & 1.00 & \\
\hline & Non-working & $27(36.0)$ & $48(64.0)$ & $1.19(0.87,1.62)$ & 0.271 \\
\hline \multirow[t]{2}{*}{ Living arrangement } & Not with family & $658(30.0)$ & $1534(70.0)$ & 1.00 & \\
\hline & With family & $260(31.6)$ & $563(68.4)$ & $1.05(0.93,1.19)$ & 0.401 \\
\hline \multirow[t]{2}{*}{ Number of children under 5} & $<3$ & $806(30.5)$ & $|84|(69.5)$ & 1.00 & \\
\hline & $\geq 3$ & $112(30.4)$ & $256(69.6)$ & $0.9(0.85,1.18)$ & 0.995 \\
\hline \multirow[t]{2}{*}{ ANC attendance } & Not attend & $416(27.5)$ & $1095(72.5)$ & 1.00 & \\
\hline & Attended $\geq 1$ & $502(33.4)$ & $1002(66.6)$ & I.2(1.08,1.35) & 0.001 \\
\hline \multirow[t]{3}{*}{ Partner's stance on ANC use } & Discourage & $18(32.1)$ & $38(67.9)$ & 1.00 & \\
\hline & No interest & $452(34.6)$ & $853(65.4)$ & I.I $(0.78, I .59)$ & 0.706 \\
\hline & Encourage & $448(27.1)$ & $1206(72.9)$ & $0.8(0.57,1.24)$ & 0.388 \\
\hline
\end{tabular}

(Continued) 
Table 6 (Continued).

\begin{tabular}{|c|c|c|c|c|c|}
\hline \multirow[t]{2}{*}{ Variable } & \multirow[t]{2}{*}{ Category } & \multicolumn{2}{|c|}{ Overall IPVP } & \multirow[t]{2}{*}{ CPR $(95 \% \mathrm{Cl})$} & \multirow[t]{2}{*}{ P-value } \\
\hline & & Yes, n (\%) & No, $n(\%)$ & & \\
\hline \multirow[t]{3}{*}{ Number of living children } & $\leq 3$ & $538(28.8)$ & |329(7|.2) & 1.00 & \\
\hline & $4-6$ & $287(32.7)$ & $591(67.3)$ & $1.13(1.01,1.28)$ & 0.037 \\
\hline & $\geq 7$ & $93(34.4)$ & $177(65.6)$ & $1.19(1.01,1.43)$ & 0.051 \\
\hline \multirow[t]{2}{*}{ Couple's pregnancy intentions } & Concordant & $78 I(29.5)$ & $1868(70.5)$ & 1.00 & \\
\hline & Discordant & $137(37.4)$ & $229(62.6)$ & $1.27(1.09,1.47)$ & 0.001 \\
\hline \multirow[t]{2}{*}{ Previous infant loss } & No & $776(31.4)$ & $1699(68.6)$ & 1.00 & \\
\hline & Yes & $142(26.3)$ & $398(73.7)$ & $0.80(0.72,0.98)$ & 0.024 \\
\hline
\end{tabular}

Abbreviations: IPVP, intimate partner violence during pregnancy; CPR, crude prevalence ratio; ANC, antenatal care.

Table 7 Adjusted Prevalence Ratio of Factors Associated with Different Forms of Intimate Partner Violence During Pregnancy, Eastern Ethiopia, 2018

\begin{tabular}{|c|c|c|c|c|c|}
\hline \multirow{2}{*}{ Variable } & \multirow[t]{2}{*}{ Category } & \multicolumn{4}{|l|}{ APR $(95 \% \mathrm{Cl})$} \\
\hline & & Psychological IPVP & Physical IPVP & Sexual IPVP & Overall IPVP \\
\hline \multirow[t]{2}{*}{ Residence } & Harar (urban) & I & I & 1 & 1 \\
\hline & Kersa (rural) & $0.68(0.53,0.87)^{*}$ & $0.81(0.54,1.22)$ & $0.4 \mathrm{I}(0.27,0.60)^{*}$ & $0.85(0.87,1.03)$ \\
\hline \multirow[t]{3}{*}{ Age group (years) } & $<20$ & 1 & 1 & I & I \\
\hline & $20-34$ & I.83 (I.34, 2.49)* & $2.00(1.25,3.18)^{*}$ & $1.08(0.75,1.56)$ & $1.56(1.23,1.98)^{*}$ \\
\hline & $\geq 35$ & $1.60(1.10,2.33)^{*}$ & $1.60(0.89,2.86)$ & $0.90(0.55,1.46)$ & $1.37(1.02,1.84)$ \\
\hline \multirow[t]{2}{*}{ Age at first marriage (years) } & $<18$ & I & I & I & I \\
\hline & $\geq 18$ & $0.99(0.86,1.13)$ & $1.05(0.85,1.29)$ & $0.98(0.77,1.23)$ & $0.94(0.84,1.05)$ \\
\hline \multirow[t]{3}{*}{ Education (women) } & No formal education & I & I & I & 1 \\
\hline & Primary/secondary & $0.83(0.70,0.99)^{*}$ & $0.55(0.4 \mathrm{I}, 0.74)^{*}$ & $0.46(0.34,0.64)^{*}$ & $0.77(0.66,0.89)^{*}$ \\
\hline & Tertiary & $0.49(0.3 \mathrm{I}, 0.78)^{*}$ & $0.05(0.01,0.23)^{*}$ & $0.20(0.09,0.45)^{*}$ & $0.36(0.24,0.53)^{*}$ \\
\hline \multirow[t]{3}{*}{ Education (partner) } & No formal & I & 1 & I & 1 \\
\hline & Primary/secondary & $0.98(0.84,1.13)$ & $0.96(0.76,1.20)$ & $0.74(0.58,0.96)^{*}$ & $0.92(0.82,1.03)$ \\
\hline & Tertiary & $0.86(0.61,1.19)$ & $1.13(0.64,1.98)$ & $0.94(0.53,1.68)$ & $0.98(0.75,1.28)$ \\
\hline \multirow[t]{4}{*}{ Marital duration (years) } & $\leq 1$ & 1 & 1 & 1 & 1 \\
\hline & $2-4$ & $0.93(0.7 \mathrm{I}, \mathrm{I} .23)$ & $0.99(0.63,1.56)$ & $1.59(0.98,2.56)$ & $1.02(0.8 \mathrm{I}, \mathrm{I} .28)$ \\
\hline & $5-10$ & $0.76(0.57,1.01)$ & $0.77(0.48,1.21)$ & $1.10(0.67,1.80)$ & $0.78(0.62,1.00)$ \\
\hline & $>10$ & $0.97(0.7 \mathrm{I}, \mathrm{I} .3 \mathrm{I})$ & $0.99(0.60,1.63)$ & $\mathrm{I} .55(0.92,2.6 \mathrm{I})$ & $0.92(0.72,1.19)$ \\
\hline \multirow[t]{3}{*}{ Household empowerment status } & Low & I & 1 & 1 & 1 \\
\hline & Medium & I.4I (I.20, I.65)* & $1.40(1.10,1.76)^{*}$ & $1.80(1.39,2.35)^{*}$ & $1.29(1.13,1.47)^{*}$ \\
\hline & High & $0.99(0.84,1.19)$ & $0.85(0.65,1.11)$ & $0.95(0.68,1.32)$ & $0.95(0.82,1.10)$ \\
\hline \multirow[t]{3}{*}{ Drinking habit (women) } & Yes, frequently & I & 1 & I & 1 \\
\hline & Yes, occasionally & $0.77(0.41,1.43)$ & $1.15(0.47,2.83)$ & $0.66(0.28,1.56)$ & $0.89(0.55,1.44)$ \\
\hline & Not at all & $0.53(0.29,0.99)^{*}$ & $0.61(0.29,1.32)$ & $0.25(0.12,0.57)^{*}$ & $0.55(0.35,0.87)^{*}$ \\
\hline \multirow[t]{3}{*}{ Drinking habit (partner) } & Yes, frequently & 1 & 1 & 1 & I \\
\hline & Yes, occasionally & $0.95(0.65,1.40)$ & $1.54(0.75,3.16)$ & $0.68(0.33,1.38)$ & $0.90(0.65,1.25)$ \\
\hline & Not at all & $0.75(0.55,1.03)$ & $0.98(0.53,1.82)$ & $1.02(0.55,1.92)$ & $0.77(0.58,1.01)$ \\
\hline
\end{tabular}

(Continued) 
Table 7 (Continued).

\begin{tabular}{|c|c|c|c|c|c|}
\hline \multirow[t]{2}{*}{ Variable } & \multirow[t]{2}{*}{ Category } & \multicolumn{4}{|l|}{ APR $(95 \% \mathrm{Cl})$} \\
\hline & & Psychological IPVP & Physical IPVP & Sexual IPVP & Overall IPVP \\
\hline Working status (women) & $\begin{array}{l}\text { Housewife } \\
\text { Working }\end{array}$ & $1.90(1.58,2.28)^{*}$ & $\begin{array}{l}\text { I } \\
2.54(I .89,3.4 I)^{*}\end{array}$ & $\begin{array}{l}\text { I } \\
3.37(2.54,4.47)^{*}\end{array}$ & $2.13(1.83,2.48)^{*}$ \\
\hline Working status (partner) & $\begin{array}{l}\text { Working } \\
\text { Not working }\end{array}$ & $\begin{array}{l}1 \\
0.80(0.56,1.14)\end{array}$ & $\begin{array}{l}\mathrm{I} \\
0.47(0.22,1.02)\end{array}$ & $0.46(0.22,0.97)^{*}$ & $0.65(0.49,0.86)^{*}$ \\
\hline Khat-chewing habit (partner) & $\begin{array}{l}\text { Never/not habitually } \\
\text { Always/habitually }\end{array}$ & $\begin{array}{l}\text { I } \\
2.05(1.59,2.67)^{*}\end{array}$ & $\begin{array}{l}\text { I } \\
3.15(1.97,5.03)^{*}\end{array}$ & $\begin{array}{l}\text { I } \\
1.47(0.98,2.18)\end{array}$ & I $1.72(1.4 \mathrm{I}, 2.1 \mathrm{I})^{*}$ \\
\hline Living arrangement & $\begin{array}{l}\text { Not with family/parent } \\
\text { With family/parents }\end{array}$ & $\begin{array}{l}\text { I } \\
0.8 \mathrm{I}(0.69,0.94)^{*}\end{array}$ & $\begin{array}{l}\text { I } \\
0.73(0.58,0.94)^{*}\end{array}$ & $\begin{array}{l}\text { I } \\
0.93(0.74,1.17)\end{array}$ & $\begin{array}{l}\text { I } \\
0.87(0.77,0.98)^{*}\end{array}$ \\
\hline Number of children under 5 & $\begin{array}{l}<3 \\
\geq 3\end{array}$ & $0.90(0.74,1.10)$ & $\begin{array}{l}\text { I } \\
1.09(0.82,1.44)\end{array}$ & $\begin{array}{l}\text { I } \\
1.15(0.84,1.56)\end{array}$ & $\begin{array}{l}\mathrm{I} \\
0.98(0.83,1.15)\end{array}$ \\
\hline ANC attendance & $\begin{array}{l}\text { Not attended } \\
\text { Attended } \geq 1\end{array}$ & $\mathrm{I} .12(0.95,1.3 \mathrm{I})$ & I.I $(0.90,1.46)$ & $\begin{array}{l}\mathrm{I} \\
1.22(0.94,1.57)\end{array}$ & $1.25(1.09,1.42)^{*}$ \\
\hline Partner's stance on ANC use & $\begin{array}{l}\text { Discouraged } \\
\text { No interest } \\
\text { Encouraged }\end{array}$ & $\begin{array}{l}\text { I } \\
1.13(0.76,1.66) \\
0.80(0.55,1.16)\end{array}$ & $\begin{array}{l}\text { I } \\
0.52(0.29,0.92)^{*} \\
0.44(0.25,0.78)^{*}\end{array}$ & $\begin{array}{l}\text { I } \\
4.90(0.66,36.4) \\
4.34(0.58,32.2)\end{array}$ & $\begin{array}{l}\text { I } \\
0.89(0.61,1.30) \\
0.68(0.47,1.00)\end{array}$ \\
\hline Number of living children & $\begin{array}{l}\leq 3 \\
4-6 \\
\geq 7\end{array}$ & $\begin{array}{l}\text { I } \\
\text { I.12(0.93, I.34) } \\
1.19(0.89,1.57)\end{array}$ & $\begin{array}{l}\text { I } \\
\text { I.05 }(0.80,1.39) \\
\text { I. } 10(0.73,1.77)\end{array}$ & $\begin{array}{l}\text { I } \\
0.77(0.58,1.03) \\
0.96(0.64,1.44)\end{array}$ & $\begin{array}{l}\text { I } \\
\text { I.04 }(0.90,1.20) \\
\text { I.05 }(0.85,1.29)\end{array}$ \\
\hline Couple's pregnancy intentions & $\begin{array}{l}\text { Concordant } \\
\text { Discordant }\end{array}$ & $1.19(1.01,1.40)^{*}$ & $\begin{array}{l}\mathrm{I} \\
\mathrm{I} .29(\mathrm{I} .0 \mathrm{I}, \mathrm{I} .66)^{*}\end{array}$ & $\begin{array}{l}\text { I } \\
0.71(0.49,1.04)\end{array}$ & $1.19(1.03,1.36)^{*}$ \\
\hline Previous infant loss & $\begin{array}{l}\text { No } \\
\text { Yes }\end{array}$ & $\begin{array}{l}\text { I } \\
0.82(0.68,0.98)^{*}\end{array}$ & $\begin{array}{l}\text { I } \\
0.79(0.60,1.03)\end{array}$ & $\begin{array}{l}\text { I } \\
0.67(0.51,0.89)^{*}\end{array}$ & $\begin{array}{l}\text { I } \\
0.82(0.7 \mathrm{I}, 0.96)^{*}\end{array}$ \\
\hline
\end{tabular}

Note: *Significant at $P<0.05$.

Abbreviations: APR, adjusted prevalence ratio; IPVP, intimate partner violence during pregnancy; ANC, antenatal care.

The study also has some limitations. The cross-sectional nature of the study makes it difficult to establish a temporal relationship between predictor and outcome variables. We also relied on self-report of potentially stigmatizing information, such as women's alcohol consumption habits during the index pregnancy and their exposure to sexual violence, which could potentially bias their responses and lead to underreporting. Moreover, there may be a reporting bias due to using women as proxy respondents regarding their husband's/ partner's pregnancy intentions towards the index pregnancy.

\section{Implications for Clinical Practice and Future Research}

The findings of this study have implications for public health policy, clinical practice and future research. As a high proportion of women experienced IPVP, early intervention through screening of IPV in the ANC setting as well during home visits is imperative. Moreover, the high proportion of acts of sexual violence during pregnancy being in the form of forceful sex (94.6\%) signals the seriousness of the problem to the women's reproductive health and calls for the integration of information and education on IPVP into the existing maternal health programs in Ethiopia. Most importantly, given the high prevalence of IPVP in Ethiopia ${ }^{14}$ and its adverse effects on the mother and fetus/newborn, ${ }^{21,54}$ the findings of this study should contribute towards the development of national policy in the protection of pregnant women against IPV. Future longitudinal studies could investigate patterns in the prevalence of IPV around the time of pregnancy to provide better information on the risk factors of IPVP. 


\section{Conclusion}

All forms of IPVP are widely prevalent in the study area, with psychological violence being the leading type, followed by physical and sexual violence. Nearly one-third (30.5\%) of studied women had experienced at least one type of IPVP. Psychological and physical violence were the most common overlapping forms of IPVP. An enormously high proportion of sexual violence acts during pregnancy $(94.6 \%)$ was in the form of forceful sex. The women's educational level, working condition and household decision-making autonomy (empowerment), place of residence, living arrangement, alcohol intake during the index pregnancy and age; their husband's/partner's stance on women's ANC use, khat-chewing habits and working conditions; and the couple's pregnancy intentions were positively or negatively associated with the varying forms of IPVP.

More efforts are needed towards early screening for IPV and the integration of IPV information in maternal health-care programs to tackle the prevailent IPVP in the study area. Specific protective measures, including the promotion of women's higher education and sensitization on IPVP through the dissemination of relevant sensitization tools, are also paramount. Such actions increase the women's and the community's awareness about the consequences of varyiious forms of IPVP and could contribute towards changing gender-inequitable norms, beliefs and practices in the community. To this end, urgent attention at all levels of societal organization, involving different stakeholders working on women's rights, policy makers and other concerned bodies, is duly needed.

\section{Abbreviations}

ANC, antenatal care; APR, adjusted prevalence ratio; CI, confidence Interval; CPR, crude prevalence ratio; HDSS, health and demographic surveillance system; IPVP, intimate partner violence in/during pregnancy; $\mathrm{PR}$, prevalence ratio; WHO, World Health Organization.

\section{Data Sharing Statement}

All data analyzed for this article are available from the corresponding author and can be obtained on reasonable request.

\section{Acknowledgments}

The authors would like to thank Haramaya University for funding this study. They are also grateful for the additional funding and technical support obtained from the African Population and Health Center (APHRC) through the African Doctoral Dissertation Research Fellowship (ADDRF) program. The authors also wish to thank the study participants, data collectors, and staff of the School of Public Health, College of Health and Medical Sciences, for their kind cooperation.

\section{Author Contributions}

All authors made substantial contributions to conception and design, acquisition of data, or analysis and interpretation of data; took part in drafting the article or revising it critically for important intellectual content; gave final approval of the version to be published; and agree to be accountable for all aspects of the work.

\section{Disclosure}

The authors declare that they have no conflicts of interest.

\section{References}

1. World Health Organization (WHO). Preventing Intimate Partner and Sexual Violence Against Women: Taking Action and Generating Evidence; 2010.

2. Garcia-Moreno C, Zimmerman C, Morris-Gehring A, et al. Addressing violence against women: a call to action. Lancet. 2015;385(9978):1685-1695. doi:10.1016/S0140-6736(14)61830-4

3. Macy RJ, Martin SL, Kupper LL, Casanueva C, Guo S. Partner violence among women before, during, and after pregnancy: multiple opportunities for intervention. Womens Health Issues. 2007;17 (5):290-299. doi:10.1016/j.whi.2007.03.006

4. Mercedes M, Lafaurie V. Intimate partner violence against women during pregnancy: a critical reading from a gender perspective. Rev Colomb Entomol. 2015;10(10):64-77.

5. Brownridge DA, Taillieu TL, Tyler KA, Tiwari A, Chan KL, Santos SC. Pregnancy and intimate partner violence: risk factors, severity, and health effects. Violence Against Women. 2011;17(7):858-881. doi:10.1177/1077801211412547

6. Taillieu TL, Brownridge DA. Violence against pregnant women: prevalence, patterns, risk factors, theories, and directions for future research. Aggress Violent Behav. 2010;15(1):14-35. doi:10.1016/j. avb.2009.07.013

7. Chisholm CA, Bullock L, Ferguson IIJEJ. Intimate partner violence and pregnancy: epidemiology and impact. Am J Obstet Gynecol. 2017;217(2):141-144. doi:10.1016/j.ajog.2017.05.042

8. Castro R, Peek-Asa C, Ruiz A. Violence against women in Mexico: a study of abuse before and during pregnancy. Am J Public Health. 2003;93(7):1110-1116. doi:10.2105/AJPH.93.7.1110

9. Islam MJ, Broidy L, Mazerolle P, Baird K, Mazumder N. Exploring intimate partner violence before, during, and after pregnancy in Bangladesh. J Interpers Violence. 2018;0886260518775753.

10. Erman B, Vural M, Karababa A, Mahmut A, Selek S. Decrease in domestic violence during pregnancy: a study from Turkey. $J$ Interpers Violence. 2014;29(2):203-216. doi:10.1177/088626051 3505147

11. World Health Organization. WHO Multi-Country Study on Women's Health and Domestic Violence Against Women: Initial Results on Prevalence. Health Outcomes and Women's Responses; 2005. 
12. Bazyar J, Safarpour H, Daliri S, Karimi A, Keykaleh MS, Bazyar M. The prevalence of sexual violence during pregnancy in Iran and the world: a systematic review and meta-analysis. J Inj Violence Res. 2018;10(2):63.

13. Shamu S, Abrahams N, Temmerman M, Musekiwa A, Zarowsky C. A systematic review of African studies on intimate partner violence against pregnant women: prevalence and risk factors. PLoS One. 2011;6(3):e17591. doi:10.1371/journal.pone.0017591

14. Alebel A, Kibret GD, Wagnew F, et al. Intimate partner violence and associated factors among pregnant women in Ethiopia: a systematic review and meta-analysis. Reprod Health. 2018;15(1):196. doi:10.11 86/s12978-018-0637-x

15. World Health Organization (WHO). Responding to Intimate Partner Violence and Sexual Violence Against Women: WHO Clinical and Policy guidelines. Geneva: World Health Organization; 2013.

16. Cokkinides VE, Coker AL, Sanderson M, Addy C, Bethea L. Physical violence during pregnancy: maternal complications and birth outcomes. Obstet Gynecol. 1999;93(5):661-666.

17. Bailey BA. Partner violence during pregnancy: prevalence, effects, screening, and management. Int J Womens Health. 2010;2:183-197. doi:10.2147/IJWH.S8632

18. Kaye DK, Mirembe FM, Bantebya G, Johansson A, Ekstrom AM. Domestic violence during pregnancy and risk of low birthweight and maternal complications: a prospective cohort study at Mulago Hospital, Uganda. Trop Med Int Health. 2006;11(10):1576-1584. doi:10.1111/j.1365-3156.2006.01711.x

19. Islam MJ, Broidy L, Baird K, Mazerolle P. Intimate partner violence around the time of pregnancy and postpartum depression: the experience of women of Bangladesh. PLoS One. 2017;12:5. doi:10.1371/ journal.pone. 0176211

20. Shamu S, Zarowsky C, Roelens K, Temmerman M, Abrahams N. Highfrequency intimate partner violence during pregnancy, postnatal depression and suicidal tendencies in Harare, Zimbabwe. Gen Hosp Psychiatry. 2016;38:109-114. doi:10.1016/j.genhosppsych.2015.10.005

21. Musa A, Chojenta C, Geleto A, Loxton D. The associations between intimate partner violence and maternal health care service utilization: a systematic review and meta-analysis. BMC Womens Health. 2019;19(1):36. doi:10.1186/s12905-019-0735-0

22. Mohammed BH, Johnston JM, Harwell JI, Yi H, Tsang KW-K, Haidar JA. Intimate partner violence and utilization of maternal health care services in Addis Ababa, Ethiopia. BMC Health Serv Res. 2017;17(1):178. doi:10.1186/s12913-017-2121-7

23. Renner LM. Intimate partner violence victimization and parenting stress: assessing the mediating role of depressive symptoms. Violence Against Women. 2009;15(11):1380-1401. doi:10.1177/1077801209346712

24. Demelash H, Nigatu D, Gashaw K. A case-control study on intimate partner violence during pregnancy and low birth weight, Southeast Ethiopia. Obstet Gynecol Int. 2015;2015.

25. Sigalla GN, Mushi D, Meyrowitsch DW, et al. Intimate partner violence during pregnancy and its association with preterm birth and low birth weight in Tanzania: a prospective cohort study. PLoS One. 2017;12:2. doi:10.1371/journal.pone. 0172540

26. Pastor-Moreno G, Ruiz-Perez I, Henares-Montiel J, Petrova D. Intimate partner violence during pregnancy and risk of fetal and neonatal death: a meta-analysis with socioeconomic context indicators. Am J Obstet Gynecol. 2019.

27. Heise LL. Violence against women: an integrated, ecological framework. Violence Against Women. 1998;4(3):262-290. doi:10.1177 1077801298004003002

28. Gashaw BT, Schei B, Magnus JH. Social ecological factors and intimate partner violence in pregnancy. PLoS One. 2018;13(3): e0194681. doi:10.1371/journal.pone.0194681

29. Abate BA, Wossen BA, Degfie TT. Determinants of intimate partner violence during pregnancy among married women in Abay Chomen district, Western Ethiopia: a community based cross sectional study BMC Womens Health. 2016;16(1):16. doi:10.1186/s12905-016-0294-6
30. Laelago T, Belachew T, Tamrat M. Prevalence and associated factors of intimate partner violence during pregnancy among recently delivered women in public health facilities of Hossana town, Hadiya zone, southern Ethiopia. Open Access Lib J. 2014;1(07):1.

31. Yimer T, Gobena T, Egata G, Mellie H. Magnitude of domestic violence and associated factors among pregnant women in Hulet Ejju Enessie District, Northwest Ethiopia. Adv Public Health. 2014;2014

32. Bifftu BB, Dachew BA, Tadesse Tiruneh B, Zewoldie AZ. Domestic violence among pregnant mothers in Northwest Ethiopia: prevalence and associated factors. Adv Public Health. 2017;2017.

33. Gebrezgi BH, Badi MB, Cherkose EA, Weldehaweria NB. Factors associated with intimate partner physical violence among women attending antenatal care in Shire Endaselassie town, Tigray, northern Ethiopia: a cross-sectional study, July 2015. Reprod Health. 2017;14 (1):76. doi:10.1186/s12978-017-0337-y

34. Lencha B, Ameya G, Baresa G, Minda Z, Ganfure G. Intimate partner violence and its associated factors among pregnant women in Bale Zone, Southeast Ethiopia: a cross-sectional study. PLoS One. 2019;14(5):e0214962. doi:10.1371/journal.pone.0214962

35. Kassa ZY, Menale AW. Physical violence and associated factors during pregnancy in Yirgalem town, South Ethiopia. CurrPediatr Res; 2016.

36. Fekadu E, Yigzaw G, Gelaye KA, et al. Prevalence of domestic violence and associated factors among pregnant women attending antenatal care service at University of Gondar Referral Hospital, Northwest Ethiopia. BMC Womens Health. 2018;18(1):138. doi:10.1186/s12905-018-0632-y

37. Yohannes K, Abebe L, Kisi T, et al. The prevalence and predictors of domestic violence among pregnant women in Southeast Oromia, Ethiopia. Reprod Health. 2019;16(1):37. doi:10.1186/s12978-019-0694-9

38. Semahegn A, Belachew T, Abdulahi M. Domestic violence and its predictors among married women in reproductive age in Fagitalekoma Woreda, Awi zone, Amhara regional state, North Western Ethiopia. Reprod Health. 2013;10(1):63. doi:10.1186/17424755-10-63

39. Haile D, Lakew Y. Khat chewing practice and associated factors among adults in Ethiopia: further analysis using the 2011 demographic and health survey. PLoS One. 2015;10(6):e0130460. doi:10.1371/journal.pone. 0130460

40. Assefa N, Berhane Y, Worku A. Pregnancy rates and pregnancy loss in Eastern Ethiopia. Acta Obstet Gynecol Scand. 2013;92(6):642647. doi:10.1111/aogs.12097

41. World Health Organization (WHO). Putting Women First: Ethical and Safety Recommendations for Research on Domestic Violence Against Women. Geneva: World Health Organization; 2001.

42. Jansen HA, Watts C, Ellsberg M, Heise L, Garcia-Moreno C. Interviewer training in the WHO multi-country study on women's health and domestic violence. Violence Against Women. 2004;10 (7):831-849. doi:10.1177/1077801204265554

43. Ellsberg M, Heise L. Researching Violence Against Women. A practical guide for researchers and activists. Paper presented at: by World Health Organization (WHO); 2005; Geneva. Marie-France Hirigoyen, 2006: Warum tust du mir das an? Gewalt in Partnerschaften. Available from: www. path.org/files/GBV_rvaw_ complete. pdf.

44. CSA (Central Statistical Agency)[Ethiopia] and ICF. Ethiopia Demographic and Health Survey 2016. Addis Ababa, Ethiopia, and Rockville, MD, USA: CSA and ICF; 2016.

45. AsJ B, Hirakata V. Alternatives for logistic regression in cross-sectional studies: an empirical comparison of models that directly estimate the prevalence ratio. BMC Med Res Methodol. 2003;3(1):21. doi:10.1186/1471-2288-3-21

46. Mahapatro M, Gupta R, Gupta V, Kundu A. Domestic violence during pregnancy in India. J Interpers Violence. 2011;26(15):29732990. doi:10.1177/0886260510390948 
47. Hoque ME, Hoque M, Kader SB. Prevalence and experience of domestic violence among rural pregnant women in KwaZulu-Natal, South Africa. S Afr J Epidemiol Infect. 2009;24(4):34-37. doi:10.1080/10158782.2009.11441360

48. Rurangirwa AA, Mogren I, Ntaganira J, Krantz G. Intimate partner violence among pregnant women in Rwanda, its associated risk factors and relationship to ANC services attendance: a population-based study. BMJ Open. 2017;7(2):e013155. doi:10.1136/bmjopen-2016-013155

49. Silva EP, Ludermir AB, VBd A, Valongueiro SA. Frequency and pattern of intimate partner violence before, during and after pregnancy. Rev Saude Publica. 2011;45:1044-1053. doi:10.1590/S003489102011005000074

50. Islam MJ, Mazerolle P, Broidy L, Baird K. Exploring the prevalence and correlates associated with intimate partner violence during pregnancy in Bangladesh. J Interpers Violence. 2017;08862 60517730029.

51. Allen M, Raghallaigh MN. Domestic violence in a developing context: the perspectives of women in Northern Ethiopia. Affilia. 2013;28 (3):256-272. doi:10.1177/0886109913495662

52. McFarlane J, Malecha A, Watson K, et al. Intimate partner sexual assault against women: frequency, health consequences, and treatment outcomes. Obstet Gynecol. 2005;105(1):99-108. doi:10.1097/ 01.AOG.0000146641.98665.b6

53. Ferdos J, Rahman MM, Jesmin SS, Rahman MA, Sasagawa T. Association between intimate partner violence during pregnancy and maternal pregnancy complications among recently delivered women in Bangladesh. Aggress Behav. 2018;44(3):294-305. doi:10. 1002/ab.21752

54. Berhanie E, Gebregziabher D, Berihu H, Gerezgiher A, Kidane G. Intimate partner violence during pregnancy and adverse birth outcomes: a case-control study. Reprod Health. 2019;16(1):22. doi:10. 1186/s12978-019-0670-4

55. Heise L, Fulu E. What works to prevent violence against women and girls. In: State of the Field of Violence Against Women and Girls: What Do We Know and What are the Knowledge Gaps; 2014.

56. Horrigan TJ, Schroeder AV, Schaffer RM. The triad of substance abuse, violence, and depression are interrelated in pregnancy. J Subst Abuse Treat. 2000;18(1):55-58. doi:10.1016/S0740-5472(99)00058-6

57. Naved RT, Persson L. Factors associated with physical spousal abuse of women during pregnancy in Bangladesh. Int Fam Plan Perspect. 2008;34:71-78. doi:10.1363/3407108

58. Fawole AO, Hunyinbo KI, Fawole OI. Prevalence of violence against pregnant women in Abeokuta, Nigeria. Aust $N$ Z J Obstet Gynaecol. 2008;48(4):405-414. doi:10.1111/j.1479-828X.2008.00868.x

59. Bohn DK, Tebben JG, Campbell JC. Influences of income, education, age, and ethnicity on physical abuse before and during pregnancy. $J$ Obstet Gynecol Neonatal Nurs. 2004;33(5):561-571. doi:10.1177/ 0884217504269009
60. Clark CJ, Silverman JG, Shahrouri M, Everson-Rose S, Groce N. The role of the extended family in women's risk of intimate partner violence in Jordan. Soc Sci Med. 2010;70(1):144-151. doi:10.1016/ j.socscimed.2009.09.024

61. Pallitto CC, Campo P. Community level effects of gender inequality on intimate partner violence and unintended pregnancy in Colombia: testing the feminist perspective. Soc Sci Med. 2005;60(10):22052216. doi:10.1016/j.socscimed.2004.10.017

62. Ansari U, Cobham B, Etim EM, et al. Insights into intimate partner violence in pregnancy: findings from a cross-sectional study in two states in Nigeria. Violence Against Women. 2017;23(4):469-481. doi:10.1177/1077801216644072

63. Heise L. What Works to Prevent Partner Violence? An Evidence Overview; 2011.

64. Vyas S, Watts C. How does economic empowerment affect women's risk of intimate partner violence in low and middle income countries? A systematic review of published evidence. J Int Dev. 2009;21 (5):577-602.

65. Rahman M, Hoque MA, Makinoda S. Intimate partner violence against women: is women empowerment a reducing factor? A study from a national Bangladeshi sample. J Fam Violence. 2011;26 (5):411-420. doi:10.1007/s10896-011-9375-3

66. Pearson E, Andersen KL, Biswas K, Chowdhury R, Sherman SG, Decker MR. Intimate partner violence and constraints to reproductive autonomy and reproductive health among women seeking abortion services in Bangladesh. Int J Gynecol Obstet. 2017;136(3):290-297. doi:10.1002/ijgo.12070

67. Shamu S, Abrahams N, Zarowsky C, Shefer T, Temmerman M. Intimate partner violence during pregnancy in $\mathrm{Z}$ imbabwe: a cross sectional study of prevalence, predictors and associations with HIV. Trop Med Int Health. 2013;18(6):696-711. doi:10.1111/tmi.12078

68. Silverman JG, Raj A. Intimate partner violence and reproductive coercion: global barriers to women's reproductive control. PLoS Med. 2014;11(9). doi:10.1371/journal.pmed.1001723

69. Ntaganira J, Muula AS, Siziya S, Stoskopf C, Rudatsikira E. Factors associated with intimate partner violence among pregnant rural women in Rwanda. Rural Remote Health. 2009;9(3):1153.

70. Devries KM, Child JC, Bacchus LJ, et al. Intimate partner violence victimization and alcohol consumption in women: a systematic review and meta-analysis. Addiction. 2014;109(3):379-391. doi:10. 1111/add.12393

71. Eaton LA, Kalichman SC, Sikkema KJ, et al. Pregnancy, alcohol intake, and intimate partner violence among men and women attending drinking establishments in a Cape Town, South Africa township. J Community Health. 2012;37(1):208-216. doi:10.1007/s10900-011-9438-7

72. Garoma S, Fantahun M, Worku A. Maternal intimate partner violence victimization and under-five children mortality in Western Ethiopia: a case control study. J Trop Pediatr. 2012;58(6):467-474. doi:10.1093/ tropej/fms018

\section{Publish your work in this journal}

The International Journal of Women's Health is an international, peerreviewed open-access journal publishing original research, reports, editorials, reviews and commentaries on all aspects of women's healthcare including gynecology, obstetrics, and breast cancer. The manuscript management system is completely online and includes a very quick and fair peer-review system, which is all easy to use. Visit http://www.dovepress.com/testimonials.php to read real quotes from published authors. 